\title{
Dynamic PGAM5 multimers dephosphorylate BCL-xL or FUNDC1 to regulate mitochondrial and cellular fate
}

\author{
Kaili Ma ${ }^{1} \cdot$ Zhi Zhang $^{2} \cdot$ Rui Chang ${ }^{1} \cdot$ Hongcheng Cheng ${ }^{1} \cdot$ Chenglong $\mathrm{Mu}^{1} \cdot$ Tian Zhao $^{1} \cdot$ Linbo Chen $^{1}$. \\ Chuanmei Zhang ${ }^{1} \cdot$ Qian Luo ${ }^{1}$. Jialing Lin $\mathbb{D}^{2,3} \cdot$ Yushan Zhu' ${ }^{1}$ Q Quan Chen ${ }^{1,4}$
}

Received: 12 March 2019 / Revised: 26 June 2019 / Accepted: 8 July 2019 / Published online: 31 July 2019

(c) The Author(s), under exclusive licence to ADMC Associazione Differenziamento e Morte Cellulare 2019

\begin{abstract}
Mitochondria are highly dynamic organelles and respond to stress by changing their fission-fusion cycle, undergoing mitophagy, or releasing apoptotic proteins to initiate cell death. The molecular mechanisms that sense different stresses and coordinate distinct effectors still await full characterization. Here, we show that PGAM5, which exists in an equilibrium between dimeric and multimeric states, dephosphorylates BCL-xL to inhibit apoptosis or FUNDC1 to activate mitofission and mitophagy in response to distinct stresses. In vinblastine-treated cells, PGAM5 dephosphorylates BCL-xL at Ser62 to restore BCL-xL sequestration of BAX and BAK and thereby resistance to apoptosis. Selenite-induced oxidative stress increases the multimerization of PGAM5, resulting in its dissociation from BCL-xL, which causes increased BCL-xL phosphorylation and apoptosis. Once freed, the more multimeric and active PGAM5 dephosphorylates FUNDC1 to initiate mitofission and mitophagy. The reciprocal interaction of PGAM5 with FUNDC1 and BCL-xL, controlled by PGAM5 multimerization, serves as a molecular switch between mitofission/mitophagy and apoptosis.
\end{abstract}

Edited by M. Piacentini

Supplementary information The online version of this article (https:// doi.org/10.1038/s41418-019-0396-4) contains supplementary material, which is available to authorized users.

\footnotetext{
$\triangle$ Jialing Lin

jialing-lin@ouhsc.edu

$\triangle$ Yushan Zhu zhuys@nankai.edu.cn

$\triangle$ Quan Chen chenq@nankai.edu.cn chenq@ioz.ac.cn

1 State Key Laboratory of Medicinal Chemical Biology, College of Life Sciences, Nankai University, Tianjin 300071, China

2 Department of Biochemistry and Molecular Biology, University of Oklahoma Health Sciences Center, Oklahoma City, OK 73126, USA

3 Stephenson Cancer Center, University of Oklahoma Health Sciences Center, Oklahoma City, OK 73126, USA

4 State Key Laboratory of Membrane Biology, Institute of Zoology, Chinese Academy of Sciences, Beijing 100101, China
}

\section{Introduction}

Mitochondria are bioenergetic and biosynthetic organelles which play a central role in cell signaling and homeostasis [1], Mitochondria are also a major source and target of reactive oxygen species $[2,3]$. In response to bioenergetic demand and oxidative stress, mitochondria change their morphology through fission and fusion. The damaged or superfluous mitochondria are selectively removed by mitophagy. If the damage is severe and the damaged mitochondria cannot be effectively removed, the mitochondrial outer membrane will be permeabilized and the released mitochondrial proteins will trigger apoptosis. Mitochondrial outer membrane permeabilization (MOMP) is regulated by BCL-2-family proteins including the antiapoptotic BCL-2 and BCL-xL and the pro-apoptotic BAX and $\mathrm{BAK}$ as well as the BH3-only proteins [4]. BCL-2/ BCL-xL inhibit MOMP by sequestering either the BH3only proteins to prevent $\mathrm{BAX} / \mathrm{BAK}$ activation or the activated $\mathrm{BAX} / \mathrm{BAK}$ to prevent oligomeric pore formation in the MOM.

Mitochondria are highly dynamic organelles that undergo cycles of fission and fusion to maintain mitochondrial networks and inheritance. The mitochondria segregated by fission are either fused back to the network, 
or engulfed by mitophagy if they were damaged. Two distinct mitophagy pathways that have been described are the PINK1/Parkin and the mitophagy receptors-mediated pathways [5]. In particular, the mitophagy receptors NIX, FUNDC1 and PHB2 interact with the autophagosome protein LC3 to mediate mitophagy [6-8].

As a mitochondrial Ser/Thr phosphatase, PGAM5 participates in multiple processes that regulate mitochondrial homeostasis. It dephosphorylates Drp1 to promote mitochondrial fission and necrosis in response to TNF- $\alpha$ [9]. It binds AIF to trigger mitophagic cell death [10]. Its deficiency suppresses the PINK1-mediated mitochondrial degeneration in Drosophila [11] and causes a Parkinson'slike phenotype and resistance to metabolic stress in mammals [12, 13]. We have shown that PGAM5 dephosphorylates FUNDC1 to promote mitophagy in response to mitochondrial stresses [14]. PGAM5 also could interact with anti-apoptotic protein BCL-xL [15]. Interestingly, the function of BCL-xL is regulated by phosphorylation of many Ser/Thr residues, including Ser49 by PLK3 [16], Thr47 and Thr115 by JNK [17], Ser73 by CDK2 [18] and Ser14 by MST1 [19]. Here, we show that different stresses regulate the multimeric state of PGAM5, which in turn regulates its interaction with BCL-xL and FUNDC1, and their phosphorylation and function. We thus reveal a molecular switch mediated by the reciprocal PGAM5 interaction with and modification of BCL-xL and FUNDC1 that controls mitochondrial morphology, mitophagy and apoptosis.

\section{Results}

\section{PGAM5 dephosphorylates BCL-xL at Ser62}

PGAM5 dephosphorylates BCL-xL peptides [20] and interacts with BCL-xL protein [15]. To determine whether PGAM5 is able to dephosphorylate BCL-xL protein in cells, we generated $B C L-x L$ knockout, PGAM5 knockout and $B C L-x L / P G A M 5$ double knockout HeLa cell lines (Fig. 1a). FLAG-BCL-xL expressed in PGAM5 knockout cells was phosphorylated after treatment with vinblastine, a chemotherapy drug inducing mitotic arrest and BCL-xL phosphorylation (Fig. 1b, c) [21, 22]. Without vinblastine BCL-xL phosphorylation was undetectable even when the cells co-expressed the kinases PINK1 or MST1 that could phosphorylate BCL-xL [16, 19, 23]. Interestingly, coexpressing PGAM5-MYC diminished the vinblastineinduced BCL-xL phosphorylation (Fig. 1d), suggesting that PGAM5 dephosphorylates BCL-xL.

Earlier studies suggest that vinblastine induces BCL-xL phosphorylation at Ser62 via JNK or CDK1 [21, 22]. Using an antibody specific to the phospho-Ser62 of BCL-xL
(Fig. S1a), we found that the phosphorylation of Ser62 of FLAG-BCL-xL was increased in PGAM5 knockout HeLa cells (Figs. 1e and S1b) or PGAM5 knockdown HepG2 cells (Fig. S1c). Moreover, wild-type PGAM5, but not the phosphatase-dead mutant (H105A), reversed the vinblastine-induced phosphorylation of FLAG-BCL-xL at Ser62 (Figs. 1f and S1d). Wild-type PGAM5 also blocked the phosphorylation of endogenous BCL-xL at Ser62 in vinblastine-treated cells, suggesting that PGAM5 targets the phospho-Ser62 of BCL-xL (Figs. 1g and S1e). To further verify that the Ser62-phosphorylated BCL-xL is a substrate of PGAM5, we isolated GFP-His-BCL-xL from PGAM5/ $B C L-x L$ knockout cells after vinblastine treatment and incubated it with purified PGAM5-His-MYC- $\Delta$ N21 protein or the phosphatase-dead mutant. The recombinant phosphatase completely dephosphorylated the BCL-xL at Ser62, whereas the phosphatase-dead mutant did not (Fig. 1h). In contrast to BCL-xL, ectopically expressed PGAM5 neither interacted with BCL-2 (Fig. S1f, g) nor reduced the vinblastine-induced BCL-2 phosphorylation (Fig. S1h). Our results demonstrate that vinblastine induces BCL-xL phosphorylation at Ser62, which is directly and specifically reversed by the phosphatase PGAM5.

\section{Dephosphorylation of BCL-xL by PGAM5 enhances its interaction with pro-apoptotic BAX/BAK proteins and anti-apoptotic activity}

To determine the impact of Ser62 phosphorylation on BCL$\mathrm{xL}$ anti-apoptotic activity, we performed a temporal analysis of HeLa cells following treatment with vinblastine. Vinblastine induced BCL-xL Ser62 phosphorylation from 24 to 36 hours, whereas caspase- 3 and PARP cleavage began to increase at 36 hours (Figs. 2a and S2a). This suggests that BCL-xL phosphorylation occurs prior to caspase activation. As expected, a pan-caspase inhibitor Z-VAD-FKM inhibited vinblastine-induced apoptosis (Fig. S2b, c). To address the functional significance of the BCL-xL phosphorylation, we expressed FLAG-tagged BCL-xL, phosphorylation mimic mutant (S62D) or nonphosphorylatable mutant (S62A) in $B C L-x L$ knockout cells. The caspase-3 or PARP cleavage data show that $B C L-x L$ knockout cells are more sensitive to apoptotic drug vinblastine than wild-type cells (Figs. 2b and S2d). Expression of nonphosphorylatable BCL-xL inhibited the apoptosis, whereas expression of wild-type or phosphorylation mimic BCL-xL had lesser effects (Figs. 2b and S2d), which were further confirmed by the Annexin V and PI staining at two different time points (Fig. 2c). A recent report showed that the phosphorylation of BCL-xL at Ser62 lowers its binding affinity for proapoptotic BCL-2-family proteins [24]. As expected, less pro-apoptotic BAX/BAK bound to the phosphorylation mimic mutant compared to the wild-type protein or the 
a

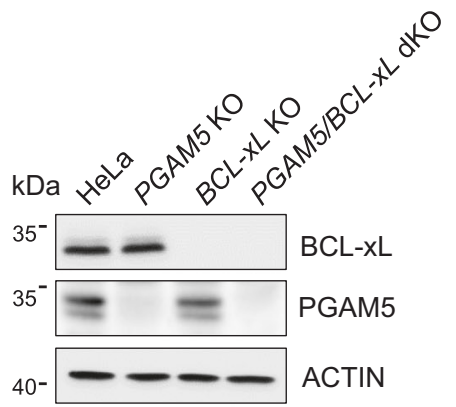

C

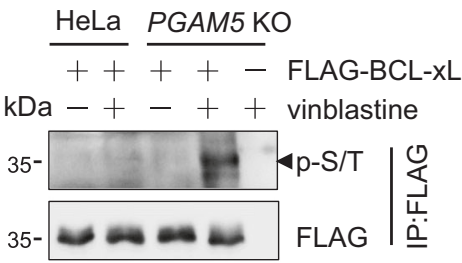

d

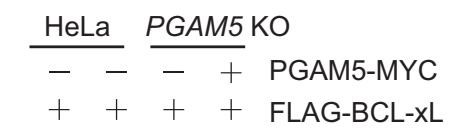

$\mathrm{kDa}-+++$ vinblastine

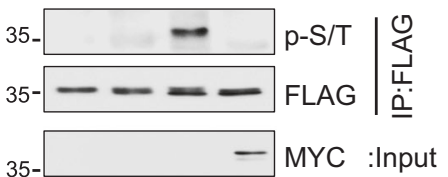

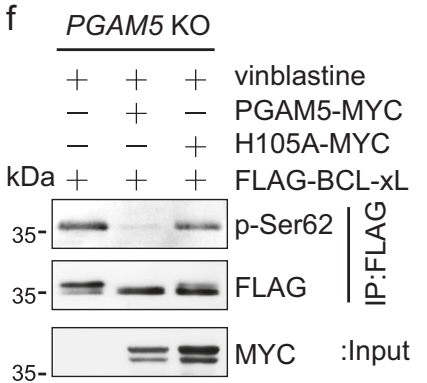

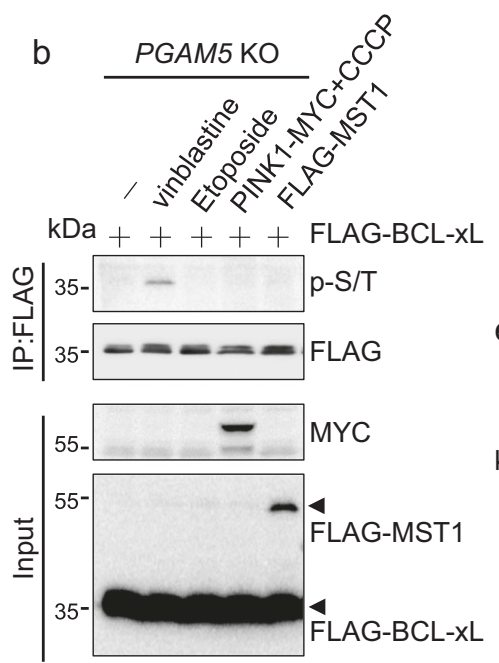

e

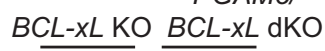

++++ FLAG-BCL-XL
kDa

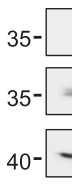

Fig. 1 The vinblastine-induced phosphorylation of BCL-xL at Ser62 is reversed by the mitochondrial phosphatase PGAM5. a Western blots of lysates from wild-type, PGAM5 knockout, $B C L-x L$ knockout or $P G A M 5 / B C L-x L$ double knockout HeLa cells using the indicated antibodies. b PGAM5 knockout HeLa cells expressing FLAG-BCL-xL with or without co-expression of PINK1-MYC or FLAG-MST1 were treated with vinblastine $(500 \mathrm{nM})$, Etoposide $(50 \mathrm{uM}), \mathrm{CCCP}(50 \mu \mathrm{M})$ or the vehicle for $24 \mathrm{~h}$. The cells were lysed for immunoprecipitation (IP) with anti-FLAG antibody and western blotting with antibodies recognizing the FLAG or MYC epitope or phospho-Ser/Thr (p-S/T). c Wild-type or PGAM5 knockout HeLa cells expressing FLAG-BCL-xL were treated with vinblastine or the vehicle for $24 \mathrm{~h}$, lysed and immunoprecipitated with anti-FLAG antibody. Total and phosphoBCL-xL were detected by western blotting with anti-FLAG or anti-pS/T antibody, respectively. d Wild-type or PGAM5 knockout HeLa cells expressing PGAM5-MYC and/or FLAG-BCL-xL were treated with vinblastine or the vehicle. The FLAG-BCL-xL in the cell lysate was enriched by IP, and the level of phospho-BCL-xL was determined by western blotting with the $\mathrm{p}-\mathrm{S} / \mathrm{T}$ antibody. e $B C L-x L$ single or $P G A M 5 / B C L-x L$ double knockout HeLa cells expressing FLAG-BCL$\mathrm{xL}$ were treated with vinblastine or the vehicle for $24 \mathrm{~h}$, lysed and

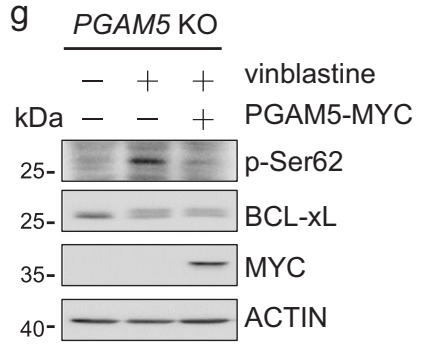

h

$$
\begin{aligned}
& +++\quad+\text { GFP-His-BCL-XL } \\
& --+- \text { PGAM5-His-MYC- } \Delta \text { N21 } \\
& -\quad-\quad+\text { H105A-His-MYC- }- \text { N21 }
\end{aligned}
$$$$
\mathrm{kDa}-+++ \text { vinblastine }
$$

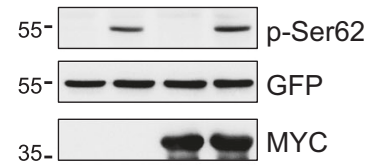

western blotted with the indicated antibodies including one that recognizes the phospho-Serine62 of BCL-xL (p-Ser62). f PGAM5 knockout HeLa cells expressing PGAM5-MYC or the phosphatasedead H105A mutant, and/or FLAG-BCL-xL were treated with vinblastine, lysed, immunoprecipitated with anti-FLAG antibody, and western blotted with the p-Ser62 antibody to detect the level of BCLxL phosphorylation at Ser62. g PGAM5 knockout HeLa cells with or without PGAM5-MYC expression were treated with vinblastine and lysed. The endogenous phospho-Ser62-BCL-xL was detected by western blotting using the p-Ser62 antibody. Note that the endogenous $\mathrm{BCL}-\mathrm{xL}$ in the vinblastine-treated cells was detected as double bands by western blotting using the anti-BCL-xL antibody. Although the nature of the double bands is unknown, it is unlikely due to the Ser62 phosphorylation, which was detected by the anti-phospho-Ser62 antibody as an extra-band above the double bands. h $P G A M 5 / B C L$ $x L$ double knockout HeLa cells expressing GFP-His-BCL-xL were treated with vinblastine and lysed. The immunoprecipitated GFP-HisBCL-xL was incubated with purified PGAM5-His-MYC- $\Delta$ N21 protein or the phosphatase-dead H105A mutant. The phospho-Ser62BCL-xL was detected by western blotting using the p-Ser62 antibody

wild-type cells (Fig. 2e). The PGAM5 knockout cells were more sensitive to vinblastine as more BAX was localized to mitochondria and more cytochrome $\mathrm{c}$ was released to the cytosol, resulting in more apoptosis (Figs. S2e, f and 2f, g). 

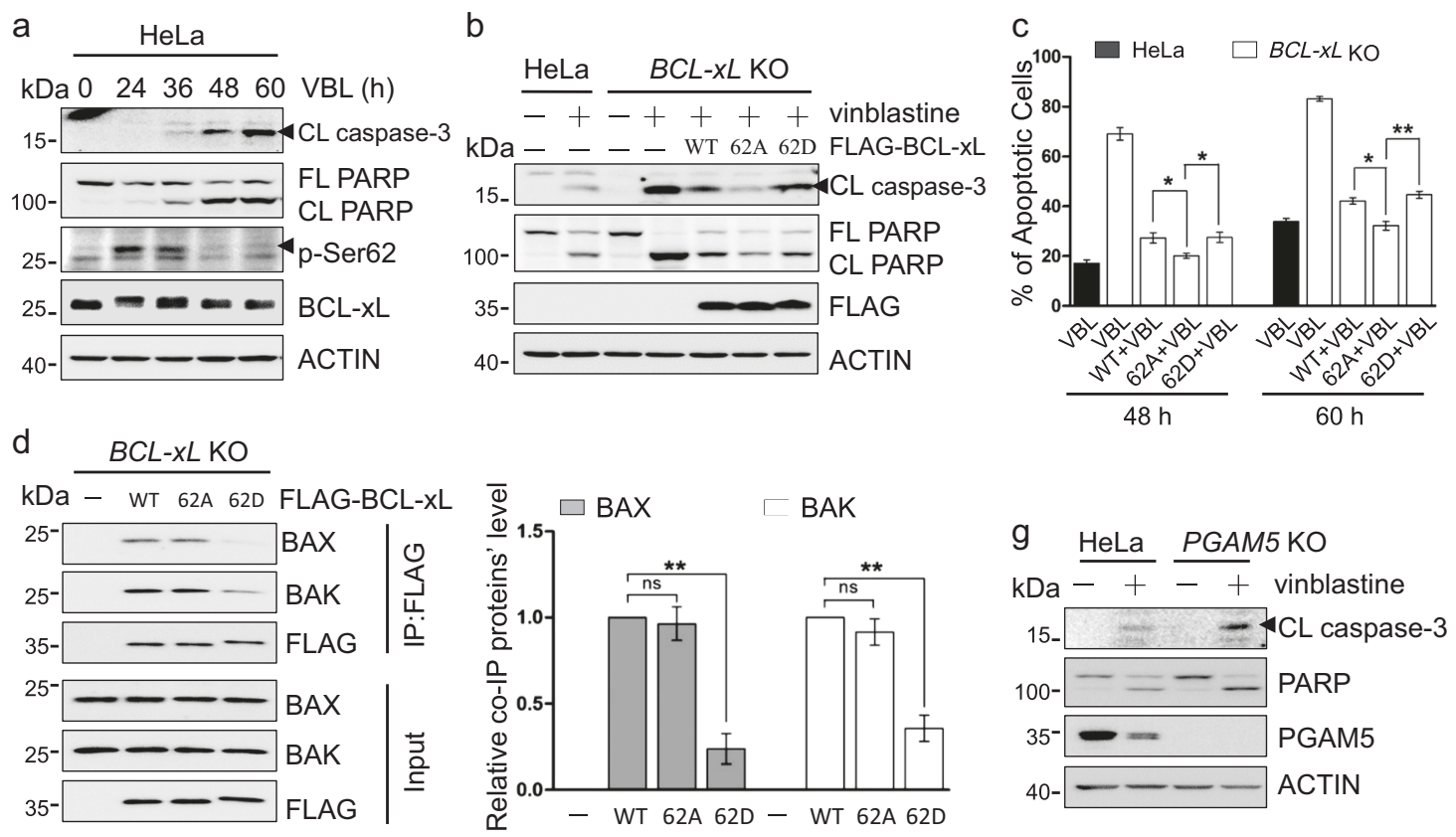

e
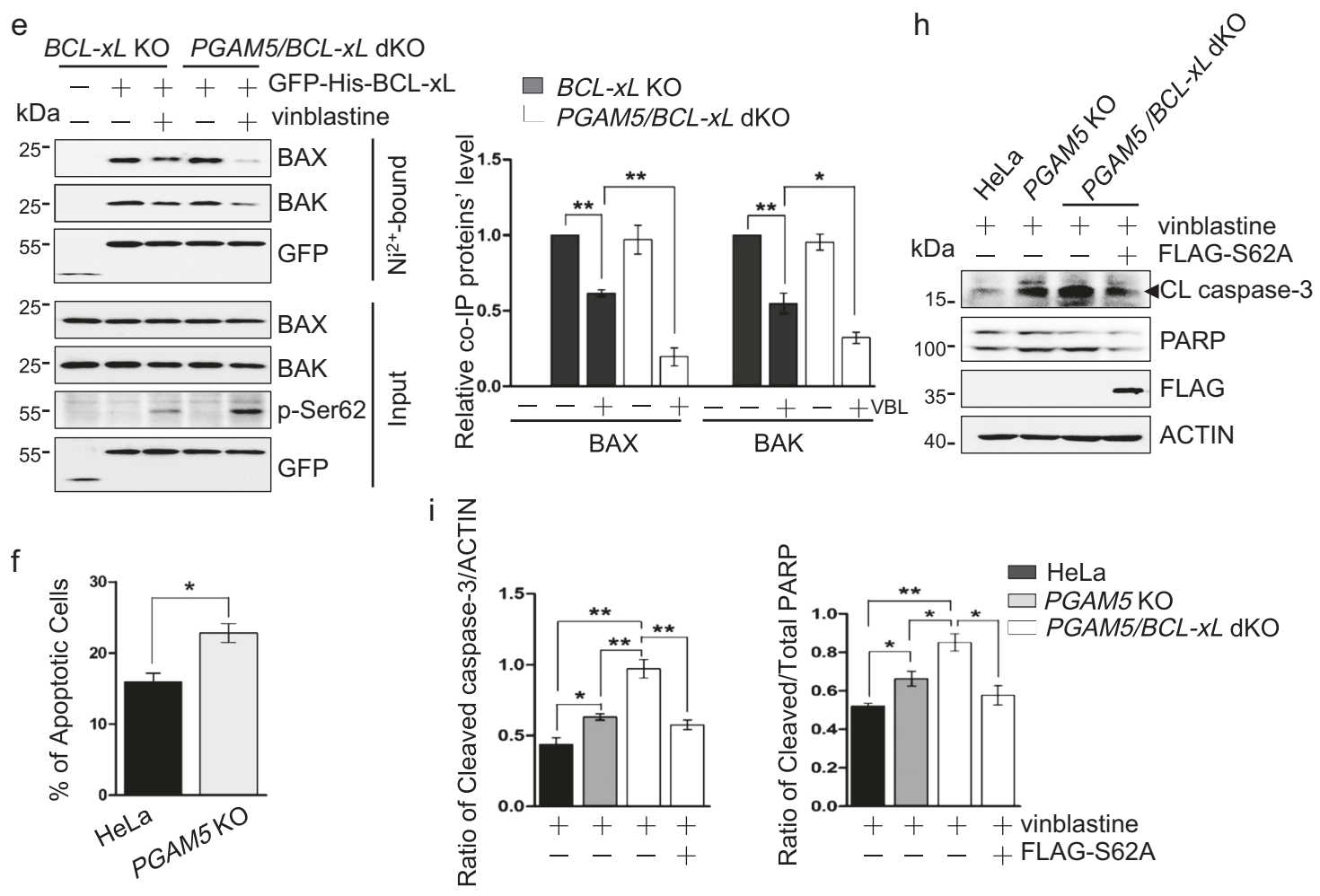

Importantly, BCL-xL deficiency made the cells more sensitive to vinblastine, whereas ectopic expression of nonphosphorylatable mutant BCL-xL significantly reduced the level of vinblastine-induced apoptosis (Fig. 2h, i).

One implication of our findings is that although PGAM5 can inhibit vinblastine-induced apoptosis by reactivating $\mathrm{BCL}-\mathrm{xL}$ using its phosphatase activity, it may promote cell death under other stress conditions through other proteins $[25,26]$. We monitored PARP cleavage in cells treated with another apoptotic stimulus, $\mathrm{H}_{2} \mathrm{O}_{2}$, or staurosporine. The results revealed that PGAM5 knockout protected cells from $\mathrm{H}_{2} \mathrm{O}_{2}$ or staurosporine, but not vinblastine (Fig. S2g, h), which suggests that PGAM5 is important for protecting cells stressed by BCL-xL phosphorylation, but not other stressors. Together, our data show that the dephosphorylation of BCL-xL by PGAM5 
Fig. 2 Dephosphorylation of BCL-xL by PGAM5 increases its antiapoptotic activity. a HeLa cells were treated with vinblastine (VBL) for the indicated times, lysed and analyzed by western blotting with the indicated antibodies. CL caspase-3, cleaved caspase-3; FL PARP, fulllength PARP; CL PARP, cleaved PARP. b HeLa or $B C L-x L$ knockout HeLa cells with or without FLAG-BCL-xL, FLAG-BCL-xL-S62A, or FLAG-BCL-xL-S62D expression were treated with vinblastine for 48 $\mathrm{h}$, lysed and analyzed by western blotting with the indicated antibodies. (c) HeLa or BCL- $x L$ knockout HeLa cells expressing FLAGBCL-xL or its mutants were treated with vinblastine for indicated times. After treatment, the cells were collected and stained with FITCannexin $\mathrm{V}$ and propidium iodide (PI), then analyzed by flow cytometry. The percentages of apoptotic cells (annexin V+/PI- and annexin $\mathrm{V}+/ \mathrm{PI}+$ ) were calculated. Data are the mean \pm SEM of three independent experiments. ${ }^{* *} p<0.01 ; * p<0.05$. d $B C L-x L$ knockout HeLa cells were transfected with FLAG-tagged wild-type BCL-xL (WT) or the S62A or S62D mutant for $24 \mathrm{~h}$, lysed and immunoprecipitated with anti-FLAG antibody. The co-immunoprecipitated endogenous BAX and BAK proteins were detected by western blotting. Right: densitometric quantification of co-immunoprecipitated BAX or BAK. Normalized to the bands from the wild type FLAGBCL-xL expressing samples. Data are the mean \pm SEM of three experiments. ${ }^{* *} p<0.01$. e $B C L-x L$ or $P G A M 5 / B C L-x L$ knockout HeLa cells were transfected with GFP-His-BCL-xL for $24 \mathrm{~h}$, treated with vinblastine for $24 \mathrm{~h}$, and lysed. The GFP-His-BCL-xL protein was isolated using $\mathrm{Ni}^{2+}$-chelating beads, eluted, and analyzed by western blotting with the indicated antibodies to detect the GFP-HisBCL-xL and the co-isolated BAX and BAK. Right: densitometric quantification of co-immunoprecipitated BAX or BAK. Normalized to the bands from the GFP-His-BCL-xL expressing untreated $B C L-x L$ knockout HeLa cells samples. Data are the mean \pm SEM of three experiments. ${ }^{*} p<0.05 ; * * p<0.01$. f Wild type or PGAM5 knockout HeLa cells were treated with $500 \mathrm{nM}$ vinblastine for $48 \mathrm{~h}$, then stained with annexin $\mathrm{V}$ and propidium iodide (PI), and analyzed by flow cytometry. Data are the mean \pm SEM of three experiments. $* p<0.05$. g HeLa or PGAM5 knockout HeLa cells were treated without or with $500 \mathrm{nM}$ vinblastine for $48 \mathrm{~h}$, lysed and analyzed by western blotting with the indicated antibodies. h HeLa, PGAM5 knockout or PGAM5/ $B C L-x L$ double knockout cells, transfected with FLAG-BCL-xL-S62A if indicated, were treated with vinblastine for $48 \mathrm{~h}$, lysed and analyzed by western blotting with the indicated antibodies. $\mathbf{i}$ The ratio of cleaved caspase- 3 to ACTIN and the ratio of cleaved PARP to total PARP in (h) were calculated, respectively. Data are the mean \pm SEM of three experiments. $* p<0.05 ; * * p<0.01$

augments the anti-apoptosis activity of BCL-xL by increasing its binding to $\mathrm{BAX} / \mathrm{BAK}$.

\section{The interaction with PGAM5 is critical for $\mathrm{BCL}-\mathrm{xL}$ dephosphorylation}

To determine whether BCL-xL dephosphorylation depends on the physical interaction with PGAM5 and the PGAM5binding site in BCL-xL, we used a photocrosslinking approach to determine which residues in BCL-xL are near PGAM5 when the proteins interact. We synthesized radioactive BCL-xL proteins, each with a photoreactive lysine ( $\varepsilon$ ANB-Lys) at a specific position. We incubated each ANB-labeled BCL-xL protein with a His $_{6}$-tagged truncated PGAM5 protein (6H-PGAM5 $\Delta$ N90) [27], and photolyzed the sample to activate the photoprobe, which can then form a covalent linkage with any PGAM5 atoms that are in close proximity. The resulting radioactive BCL-xL-PGAM5 photoadduct was separated from the unreacted BCL-xL protein using SDS-PAGE and visualized by phosphorimaging. When the $\varepsilon$ ANB-Lys was used to replace P82 in the disordered loop between $\alpha$-helices 1 and $2(\alpha 1$ and $\alpha 2)$ where the phosphorylation site (S62) is located, a BCL-xLPGAM5-specific photoadduct with the molecular weight close to the heterodimer was detected (Fig. 3a, lane 4). This photoadduct was not formed when either the ANB probe, the light (hv) or the $6 \mathrm{H}-\mathrm{PGAM} 5 \Delta \mathrm{N} 90$ was omitted (lanes 3 , 5 , or 6), or when a BCL-xL mutant not containing any lysine including the photoreactive lysine was used (lane 2). The BCL-xL-PGAM5-specific photoadduct was also formed when the R6 or K16 in the BH4 domain/ $\alpha 1$ or the E193 in the BH2 domain/ $\alpha 8$ was replaced by $\varepsilon$ ANB-Lys (Fig. 3a, lanes 8, 12, and 16) but not in the control reactions that lack the ANB probe, the light or the $6 \mathrm{H}-\mathrm{PGAM} 5 \Delta \mathrm{N} 90$. These data suggest that the PGAM5 binding site in BCL-xL is not limited to the disordered loop where the substrate phospho-S62 is located but extends to the folded regions including the $\mathrm{BH}$ domains (Fig. 3b). Moreover, PGAM5 contains a putative $\mathrm{BH} 3$ domain that may interact with the canonical BH3-binding groove in BCL-xL [28], and the $\mathrm{BH} 3$ domain in BCL-xL is critical to the interaction [29]. Therefore, we propose a model for BCL-xL/PGAM5 binding (Figs. 3b and S3a). Thus, the phospho-S62 in the disordered loop of BCL-xL binds to the active site in the PGAM5 phosphatase; the canonical BH3-binding groove formed by the $\mathrm{BH} 1$ to 3 domains binds to the $\mathrm{BH} 3$ domain of PGAM5; and the BH4 domain binds to an unknown region of PGAM5.

To test our model, we mutated residues within the potential PGAM5-binding sites in BCL-xL: R6D in BH4; G138A and R139D in BH1; and D189N and E193Q in BH2 domains. Immunoprecipitation analysis of cells expressing each mutant revealed that R6D and R139D mutations weakened the BCL-xL interaction with PGAM5, while other mutations did not (Figs. 3c and S3b). In vinblastinetreated cells, more S62-phosphorylation was detected for R6D and R139D mutants than wild-type BCL-xL or other mutants (Fig. 3d, e), suggesting that BCL-xL interaction with PGAM5 is important for the dephosphorylation by PGAM5. Consistent with our previous report [30], G138A or R139D mutation in the BH3-binding groove abolished the BCL-xL binding to BAX/BAK in untreated cells, whereas R6D mutation did not (Fig. 3c). Vinblastine treatment caused more caspase activation in cells expressing R6D than those expressing wild-type BCL-xL (Figs. 3f and $\mathrm{S} 3 \mathrm{c})$. This is consistent with lower levels of BAX/BAK binding to R6D mutant, which was caused by higher levels of S62-phosphorylation of the mutant than the wild-type BCL-xL (Fig. S3d, e). Furthermore, similar levels of 

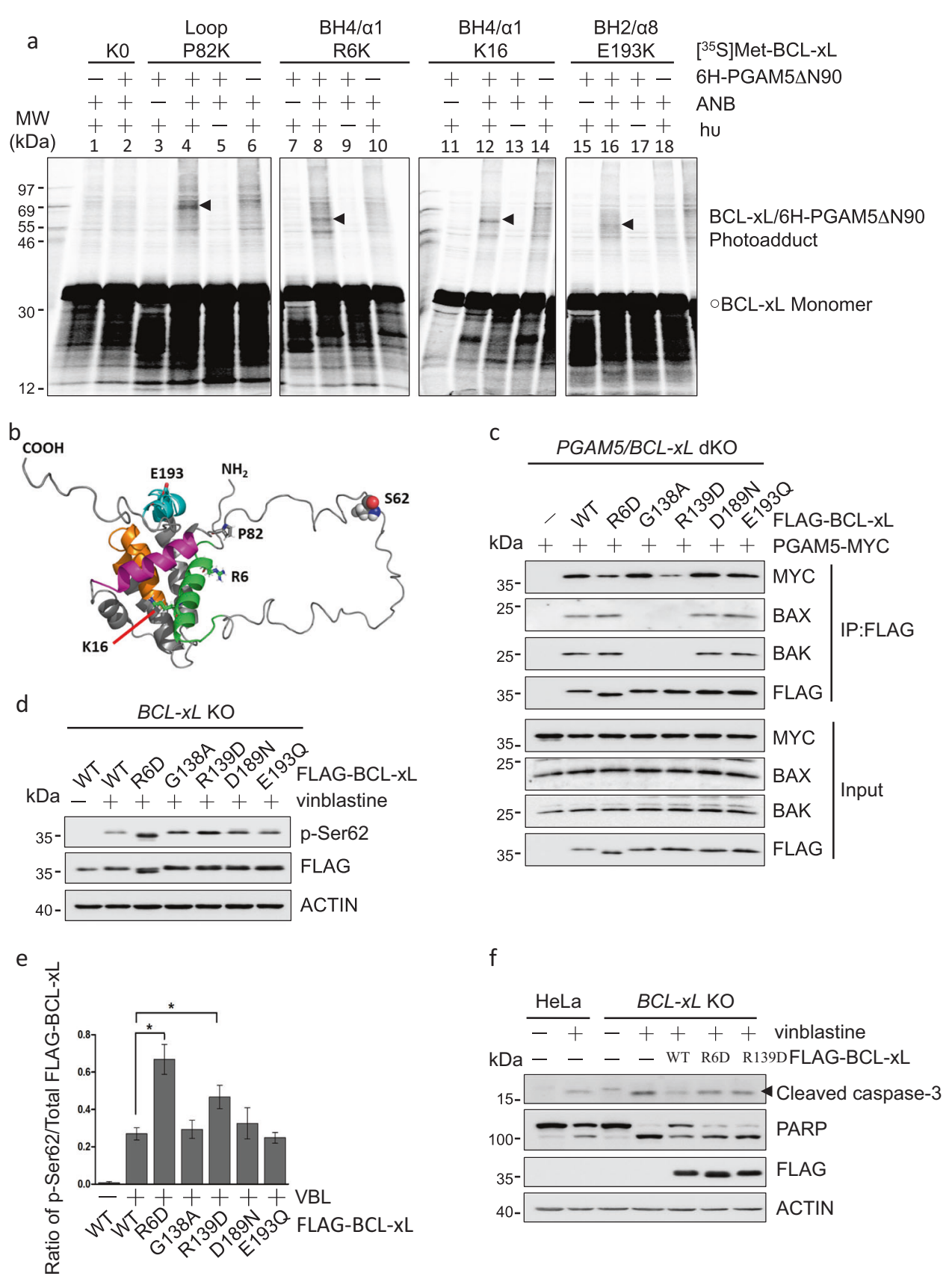

caspase activation occurred in vinblastine-treated R6D or R139D-expressing cells (Fig. 3f). Therefore, the interaction of PGAM5 with BCL-xL is critical for BCL-xL dephosphorylation and anti-apoptotic function.

\section{Selenite increases PGAM5 multimerization to decrease its interaction with and dephosphorylation of BCL-xL}

Although mitotic arrest increases BCL-xL phosphorylation, thus reducing its anti-apoptotic activity, PGAM5 binds

BCL-xL and dephosphorylates it to counter this stress and protect cells. However, an additional stress may tip the balance to augment cell death. Indeed, selenite, a reagent that targets mitochondria and triggers ROS production, enhanced the vinblastine-induced phosphorylation of BCL-xL (Figs. 4a and S4a), although selenite alone did not induce BCL-xL phosphorylation (Fig. 4b). Moreover, selenite failed to enhance the phosphorylation of BCL-xL in the absence of PGAM5, suggesting that selenite inhibited the dephosphorylation of BCL-xL by PGAM5 (Fig. 4c, d). In accordance with the increased phosphorylation of BCL-xL, 
Fig. 3 The interaction of PGAM5 with BCL-xL is critical for dephosphorylation of BCL-xL. a Photocrosslinking reaction of $\left[{ }^{35} \mathrm{~S}\right]$ Met-labeled BCL-xL proteins, each with the indicated single Lys in the indicated domain replaced by an $\varepsilon$ ANB-Lys, to His $_{6}$-tagged PGAM5 protein lacking the N-terminal 90 residues $(6 \mathrm{H}-$ PGAM $5 \Delta$ N90). The control reactions omitted the ANB probe, the light (hv) that activates the ANB probe, or the PGAM5 protein. Crosslinked products were analyzed by SDS-PAGE, alongside with another control reaction containing a Lys-null BCL-xL protein (K0). Radioactive proteins on the gel were detected by phosphor-imaging. The circle indicates the $\left[{ }^{35} \mathrm{~S}\right]$ Met-labeled BCL-xL monomer and arrowheads indicate the BCL-xL/6H-PGAM5 $\Delta$ N90 photoadducts. The molecular weights (MW) of radioactive protein standards are indicated in $\mathrm{kDa}$ on the left side. $\mathbf{b}$ The BCL-xL structure, generated from PDB entry 1LXL [39] using the PyMOL program. The $\mathrm{NH}_{2}$ and $\mathrm{COOH}$ termini are labeled. The $\mathrm{BH} 1,2,3$ and 4 domains are colored in orange, cyan, magenta and green, respectively. The residues that were replaced by $\varepsilon$ ANB-Lys in the photocrosslinking experiment are shown in stick form. The residue S62, which is located in the disordered loop and can be dephosphorylated by PGAM5, is shown in sphere form. c PGAM5/BCL- $x L$ double knockout HeLa cell lines were co-transfected with PGAM5-MYC and FLAG-BCL-xL (WT) or the arginine-6 to aspartic acid (R6D), glycine-138 to alanine (G138A), arginine-139 to aspartic acid (R139D), aspartic acid-189 to asparagine (D189N), or glutamic acid-193 to glutamine (E193Q) mutant as indicated for $24 \mathrm{~h}$. The cells were lysed and immunoprecipitated with anti-FLAG antibody. Co-immunoprecipitated PGAM5-MYC and endogenous BAX and BAK were detected by immunoblotting. d $B C L-x L$ knockout HeLa cells were transfected with FLAG-BCL-xL or the indicated mutants for $24 \mathrm{~h}$, treated with $500 \mathrm{nM}$ vinblastine for $24 \mathrm{~h}$, lysed and analyzed by immunoblotting with the indicated antibodies. $\mathrm{e}$ The ratio of $\mathrm{p}-$ Ser62 to total WT or mutant FLAG-BCL-xL were calculated from the respective band intensities in the blots in (d). Data are the mean \pm SEM of three experiments. ${ }^{*} p<0.05$. f HeLa or $B C L-x L$ knockout HeLa cells expressing FLAG-BCL-xL (WT, R6D or R139D) were treated with vinblastine for $48 \mathrm{~h}$, lysed and analyzed by immunoblotting with the indicated antibodies

less $\mathrm{BAX} / \mathrm{BAK}$ was bound with BCL-XL in selenite-treated cells (Fig. S4b, c). Short-term treatment with selenite affected neither the protein level of BCL-xL and PGAM5 in cells nor the PGAM5 phosphatase activity in vitro (Figs. 4e and S4d). However, co-IP analysis revealed that the interaction between BCL-xL and PGAM5 was significantly attenuated by selenite treatment (Figs. $4 \mathrm{f}$ and $\mathrm{S} 4 \mathrm{e}$ ).

Interestingly, the interaction of BCL-xL and PGAM5 still decreased in the selenite-treated cells even without vinblastine (Fig. S4f). This implies that mitochondrial oxidative stress may alter the BCL-xL/PGAM5 interaction independent of BCL-xL phosphorylation. Structural analyses show that PGAM5 can transform from a dimer to a dodecamer [20, 27, 31]. Intriguingly, BCL-xL underwent photocrosslinking to PGAM5 $\Delta$ N90, which forms homodimers, but not to PGAM5 $\Delta$ N54, which forms higher-order multimers (Figs. $4 \mathrm{~g}$ and $\mathrm{S} 4 \mathrm{~g}$ ). PGAM5 $\Delta$ N54, but not $\Delta$ N90, contains a highly conserved motif, WDPNWD, located in the N-terminal region of PGAM5 and required for assembly of the dodecameric complex in vitro [20, 27]. Using a WDPNWD-deletion mutant to verify whether this motif is important for PGAM5 oligomerization in cells, we found that wild-type PGAM5 formed dimers and several higher-order multimers, whereas the mutant is detected only as monomers and dimers (Fig. S4h). Furthermore, selenite enhanced the homotypic interaction of PGAM5, as more PGAM5 multimers and fewer dimers were detected in the selenite-treated cells compared to the control cells (Fig. 4h, i). Together, these results suggest a model in which selenite increases PGAM5 multimerization, reducing its association with and dephosphorylation of BCL-xL.

\section{PGAM5 dissociates from BCL-xL to promote mitochondrial fission and mitophagy through the FUNDC1 pathway}

We reported that PGAM5 could dephosphorylate FUNDC1 to promote mitochondrial fission and mitophagy, and this was inhibited by BCL-xL in the absence of stress [14, 29, 32]. Here, we observed that selenite significantly enhanced the dephosphorylation of FUNDC1 at Ser13, which was blocked by PGAM5 knockout (Figs. 5a and S5a). In comparison, neither selenite nor PGAM5 knockout affected the phosphorylation of DRP1 at Ser637 and Ser616 (Figs. 5a and S5a). In the presence of selenite, the PGAM5BCL-xL interaction decreased, whereas the PGAM5FUNDC1 interaction increased (Fig. 5b, c). As expected, dephosphorylation of FUNDC1 caused mitochondrial localization of DRP1 as well as mitochondrial fission (mitofission) shortly after selenite addition, and both were blocked by FUNDC1 knockout (Figs. 5d and S5d, b, c). Similarly, PGAM5 knockout prevented mitofission following selenite treatment (Figs. 5d and S5d), suggesting that the selenite-induced mitofission is associated with the dephosphorylation of FUNDC1 by PGAM5.

Since FUNDC1 is a mitophagy receptor [7], we examined the role of FUNDC1 in selenite-induced mitophagy. The mitochondrial proteins TIMM23 and TOMM20 and mitophagy adaptor SQSTM1 were degraded when cells were treated with selenite for 12 hours. Their degradation, a biochemical hallmark of mitophagy, was reduced by FUNDC1 knockout (Figs. 5e and S5e) or by bafilomycin A1, a lysosomal ATPase inhibitor (Fig. 5e). Similarly, PGAM5 knockout prevents selenite-induced FUNDC1 dephosphorylation and mitophagy (Figs. 5f and S5f). These results indicate that in response to selenite, the PGAM5 dimer dissociates from BCL-xL, multimerizes, and enhances mitofission and mitophagy through dephosphorylation of FUNDC1.

\section{Reciprocal interaction of PGAM5 with BCL-xL and FUNDC1 dictates mitochondrial and cellular fate}

We have shown that PGAM5 dephosphorylates BCL-xL at Ser62 to enhance its interaction with pro-apoptotic BAX/ 


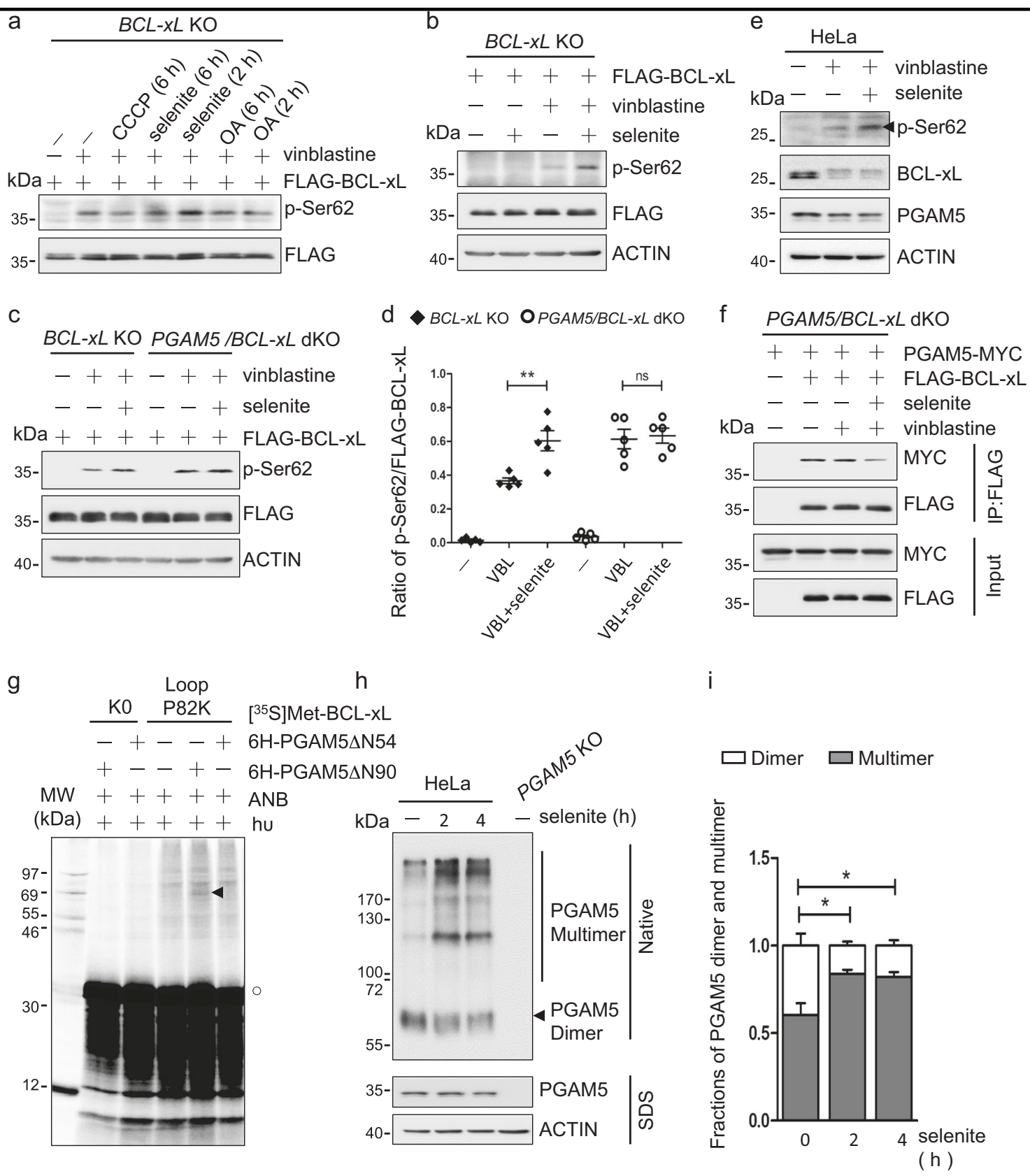

BAK, thereby preventing apoptosis. On the other hand, PGAM5 dephosphorylates FUNDC1 to promote mitophagy, an early response to mitochondrial stress that can also prevent apoptosis. A fundamental question is whether the reciprocal interaction of PGAM5 with BCL-xL and FUNDC1 serves as a molecular switch to determine mitochondrial and cellular fate in response to mitochondrial stress. We took further advantage of vinblastine and selenite to manipulate the protein phosphorylation status. FUNDC1, which is phosphorylated by CK2 in untreated cells at Ser13 [14], was dephosphorylated with selenite and/or vinblastine treatment (Fig. 6a). In contrast, the dephosphorylation of BCL-xL, which is phosphorylated at Ser62 in vinblastinetreated cells, was inhibited by selenite (Fig. 6a). Moreover, apoptotic markers were increased in cells treated with a combination of selenite and vinblastine (Figs. 6b and S6a). Previous reports suggest that selenite perturbs the redox system and generates ROS, leading to mitofission, mitophagy and apoptosis $[32,33]$. To test whether mitochondrial ROS (mito-ROS) is important for the selenite-modulated dephosphorylation of BCL-xL or FUNDC1 by PGAM5, we used MitoSox staining and found that selenite increased mito-ROS, which was reversed by mito-ROS scavenging agent MitoQ (Fig. S6b). MitoQ blocked FUNDC1 dephosphorylation while enhancing BCL-xL dephosphorylation (Figs. 6c and S6c), likely by modulating the PGAM5 interaction with BCL-xL and FUNDC1. Indeed, MitoQ strongly reversed the effects of selenite, including the 
Fig. 4 Selenite induces PGAM5 multimerization to inhibit PGAM5 interaction with and dephosphorylation of BCL-xL. a BCL- $x L$ knockout HeLa cells expressing FLAG-BCL-xL were treated with $500 \mathrm{nM}$ vinblastine for $24 \mathrm{~h}$ and $10 \mu \mathrm{M} \mathrm{CCCP}, 5 \mu \mathrm{M}$ selenite or $50 \mathrm{nM}$ oligomycin and $500 \mathrm{nM}$ antimycin (OA) for the indicated times, lysed and analyzed by immunoblotting with the indicated antibodies. b $B C L$ $x L$ knockout HeLa cells expressing FLAG-BCL-xL were treated with $500 \mathrm{nM}$ vinblastine for $24 \mathrm{~h}$ and/or $5 \mu \mathrm{M}$ selenite for $2 \mathrm{~h}$, lysed and analyzed by the indicated antibodies. c $B C L-x L$ and $P G A M 5 / B C L-x L$ knockout HeLa cells expressing FLAG-BCL-xL were treated without or with $5 \mu \mathrm{M}$ selenite for $2 \mathrm{~h}$ and $500 \mathrm{nM}$ vinblastine for $24 \mathrm{~h}$, lysed and analyzed by the indicated antibodies. $\mathbf{d}$ The ratio of p-Ser62 to total FLAG-BCL-xL were calculated from the respective band intensities in the respective blots in (c) measured by ImageJ. Data from five experiments and the mean \pm SEM are shown. ${ }^{*} p<0.01$. e HeLa cells were treated with or without selenite for $2 \mathrm{~h}$ and vinblastine for $24 \mathrm{~h}$, lysed and analyzed by immunoblotting with the indicated antibodies. f $P G A M 5 / B C L-x L$ double knockout HeLa cells expressing FLAGBCL-xL and/or PGAM5-MYC were treated with vinblastine $(500 \mathrm{nM}$, $24 \mathrm{~h})$ and selenite $(5 \mu \mathrm{M}, 2 \mathrm{~h})$ as indicated, lysed and immunoprecipitated with anti-FLAG antibody. The co-precipitated PGAM5-MYC was detected by immunoblotting with anti-MYC antibody. g Photocrosslinking reaction of a $\left[{ }^{35} \mathrm{~S}\right] \mathrm{Met}-\mathrm{BCL}-\mathrm{xL}$ protein, with the single Lys (P82K) in the loop domain replaced by an eANB-Lys, to a $6 \mathrm{H}-$ PGAM5 protein lacking the $\mathrm{N}$-terminal 54 or 90 residues $(\Delta \mathrm{N} 54$ or $\Delta \mathrm{N} 90)$. The products were analyzed by SDS-PAGE, alongside control reactions containing a Lys-null BCL-xL protein $(\mathrm{K} 0)$ or omitting the PGAM5 protein. Radioactive proteins on the gel were detected by phosphor-imaging. The circle indicates the $\left[{ }^{35} \mathrm{~S}\right]$ Met-labeled BCL-xL monomer and the arrowhead indicates the BCL-xL/6H-PGAM5 $\Delta$ N90 photoadduct. Molecular weights (MW) of protein standards are shown in $\mathrm{kDa}$ on the left side. $\mathbf{h}$ HeLa and PGAM5 knockout HeLa cells were treated with or without selenite for the indicated times, lysed, analyzed by native PAGE or denaturing (SDS) PAGE and immunoblotted with the indicated antibodies. $\mathbf{i}$ The fractions of PGAM5 dimer and multimer were determined from the intensities of the respective bands in the native gel immunoblot in (h) measured by ImageJ. Data are the mean \pm SEM of three experiments. ${ }^{*} p<0.05$

decreased BCL-xL/PGAM5 interaction, the increased FUNDC1/PGAM5 interaction, and the PGAM5 multimerization (Figs. 6d, e and S6d). As expected, selenite inhibited BCL-xL dephosphorylation, resulting in less BAX/BAK bound to BCL-xL; this effect was also rescued by MitoQ (Fig. S6e). Consequently, MitoQ inhibited the selenite-induced apoptosis (Fig. S6f). These results together suggest that selenite-induced mitochondrial oxidative stress inhibits dephosphorylation of BCL-xL by PGAM5 to promote apoptosis.

Interestingly, FUNDCl knockout attenuated the vinblastine- and selenite-induced cytochrome $\mathrm{c}$ release and apoptosis (Fig. 6f, g). Moreover, FUNDCl knockout cells expressing FUNDC1-MYC were obviously more susceptible to apoptosis induced by vinblastine and selenite (Fig. 6h). This seems contradictory to the anti-apoptotic role of FUNDC1-dependent mitophagy. Since previous studies show that inhibition of mitofission partially prevents the release of cytochrome c [34], we reasoned that FUNDC1-dependent mitofission might participate in the selenite-mediated aggravation of apoptosis in vinblastinetreated cells. Treating cells with selenite or vinblastine or their combination, we found that vinblastine failed to induce FUNDC1 dephosphorylation, but selenite increased FUNDC1 dephosphorylation, the FUNDC1/DRP1 interaction, and mitofission in the presence of vinblastine (Fig. S6g-i). In summary, selenite causes mitochondrial oxidative stress which exacerbates vinblastine-induced apoptosis by promoting FUNDC1 dephosphorylation by PGAM5. This in turn induces mitochondrial fission while also inhibiting the dephosphorylation of BCL-xL by PGAM5, thus increasing BAX/BAK-mediated MOMP.

\section{Discussion}

It is known that different stresses trigger different mitochondrial and cellular responses, but the mechanisms that sense the stresses and coordinate effectors to execute graded responses are poorly understood. Our study sheds light on these mechanisms. We show that PGAM5, which exists in an equilibrium between dimeric and oligomeric states that can sense stress, dephosphorylates either BCL-xL to "switch off" apoptosis or FUNDC1 to "switch on" mitofission and mitophagy for survival (Fig. 7). Our data suggest that in unstressed cells, BCL-xL is nonphosphorylated and strongly interacts with pro-apoptotic molecules such as BAX/BAK to promote cell survival [24]. BCL-xL also interacts with PGAM5 to inhibit FUNDC1 dephosphorylation and subsequent mitophagy [29]. However, the stress from the mitotic arrest induced by vinblastine likely activates the kinases CDK1 and/or JNK [21, 22], which phosphorylate BCL-xL to reduce its interaction with BAX/ BAK, thereby promoting apoptosis. To mitigate apoptosis, the phosphatase PGAM5 reverses the BCL-xL phosphorylation. Phospho-BCL-xL is an atypical substrate of PGAM5 as it interacts with PGAM5 through sites other than the phospho-site, and can therefore sequester the phosphatase even after dephosphorylation. Moreover, the PGAM5 dimer bound by BCL-xL has lower catalytic activity than the multimer, which does not bind BCL-xL [20]. This further increases the retention time of BCL-xL on PGAM5. Therefore, BCL-xL may function as a suicide inhibitor of PGAM5, preventing it from acting on another substrate (phospho-FUNDC1) in mitotically arrested cells.

Our data indicate that PGAM5 may serve as a sensor for oxidative stress caused by selenite to determine mitochondrial and cellular fates. Selenite-induced oxidative stress alters the multimeric status of mitochondrial PGAM5, thus freeing PGAM5 from BCL-xL (Figs. 4h, 5b and 7). This change is strongly inhibited by MitoQ, a potent scavenger that diminishes mito-ROS (Fig. 6d, e). It is possible that mito-ROS directly target PGAM5, likely at the cysteine 
a

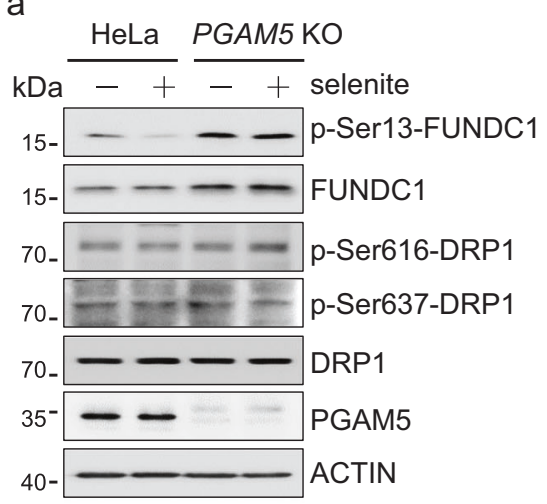

b

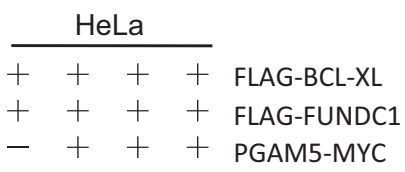

$\mathrm{kDa}-0 \quad 26$ selenite $(\mathrm{h})$

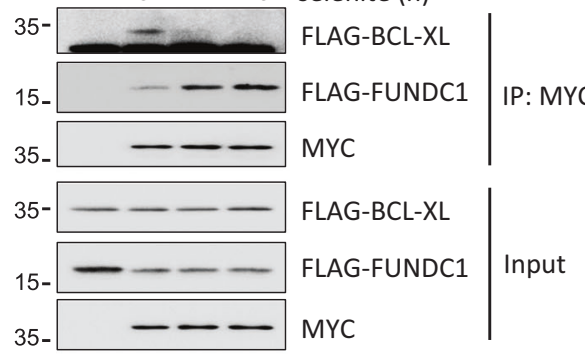

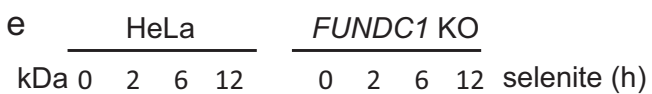

55- - - - - SQSTM1

25- $--\cdots-\cdots$ TIMM23

$15-\ldots-\ldots$ TOMM20

15-

FUNDC1

15- =-ー =ニニ- ${ }_{\text {LC3-II }}^{\text {LC3-I }}$

40-—- - - ACTIN

55- - - _ _ SQSTM1

25- $-\ldots-\ldots$ TIMM23

$15-\square-\ldots$ TOMM20

15- LC LC LC3-I

40- - - - - ACTIN
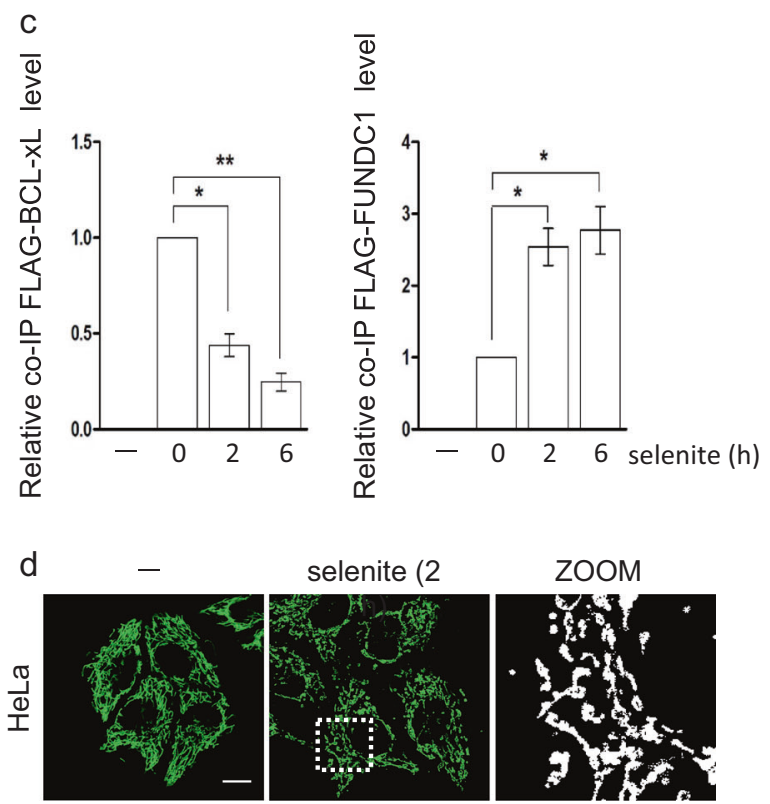

selenite $(2$

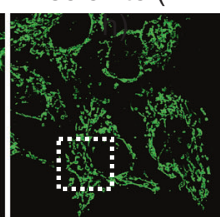

ZOOM
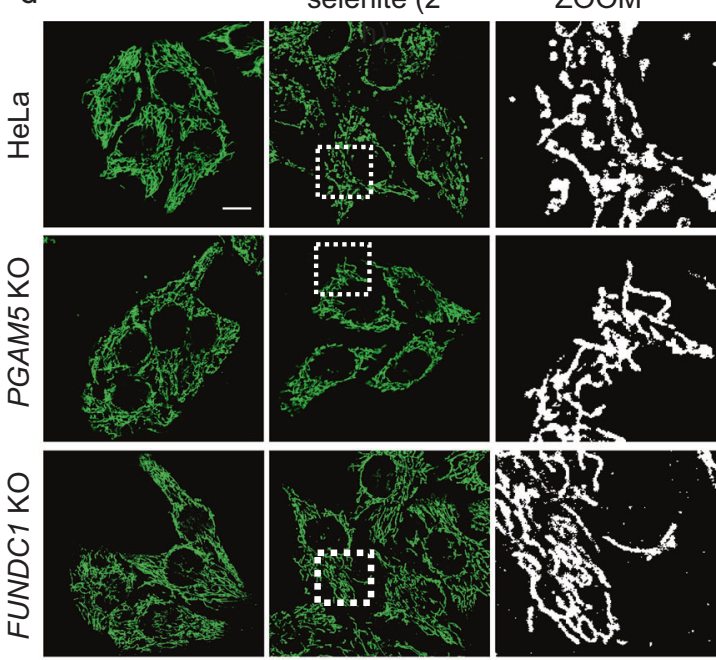

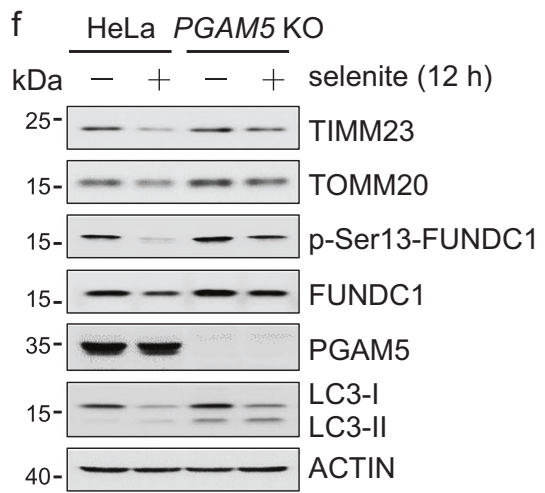

residues [35, 36], to induce oligomerization. Indeed, our unpublished data show that mutations of cysteines 12 and 229 reduce the oligomerization in response to selenite treatment. Thus, the cell responds to these distinct stresses by altering the multimerization state of PGAM5, which in turn alters the interaction of PGAM5 with BCL-xL and
FUNDC1 and hence their phosphorylation state. This balance modulates mitochondrial integrity, morphology and recycling, and ultimately makes a life-or-death decision for the cell.

When the cell experiences both mitotic and oxidative stresses, the balance is tipped toward death. The underlying 
Fig. 5 PGAM5 dephosphorylates FUNDC1 to regulate mitochondrial fission and mitophagy in response to selenite. a HeLa and PGAM5 knockout HeLa cells were treated with selenite or vehicle for $2 \mathrm{~h}$, lysed and analyzed by immunoblotting with the indicated antibodies. b HeLa cells were transfected with FLAG-BCL-xL, FLAG-FUNDC1 and PGAM5-MYC, treated with selenite for the indicated times, lysed and immunoprecipitated with anti-MYC antibody. The co-precipitated FLAG-BCL-xL and FLAG-FUNDC1 were detected by immunoblotting with anti-FLAG antibody. c Densitometric quantification of coimmunoprecipitated FLAG-BCL-xL or FLAG-FUNDC1 in (b). Normalized to the band from the untreated samples. Data are the mean \pm SEM of three experiments. $* p<0.05$; $* * p<0.01$. d HeLa and FUNDC1 or PGAM5 knockout HeLa cells were treated with selenite for $2 \mathrm{~h}$, fixed and immunostained with a fluorescent dye-conjugated antibody against the mitochondrial protein cytochrome $\mathrm{c}$ (green). Confocal fluorescence images of the cells are shown (scale bar, 10 $\mu \mathrm{m}$.) e HeLa and FUNDC1 knockout HeLa cells were treated with 5 $\mu \mathrm{M}$ selenite in the absence or presence of bafilomycin A1 (BA1) for the indicated times, lysed and analyzed by immunoblotting with the indicated antibodies. Asterisk indicates a nonspecific band detected by the anti-Ser13-FUNDC1 antibody. f HeLa and PGAM5 knockout HeLa cells were treated with $5 \mu \mathrm{M}$ selenite or vehicle for $12 \mathrm{~h}$, lysed and analyzed by immunoblotting with the indicated antibodies

molecular mechanism we revealed here is that BCL-xL loses the ability to bind to PGAM5 and thus loses the chance to be reactivated by dephosphorylation to inhibit BAX/BAK-dependent apoptosis in the mitotically arrested and oxidized cell. Furthermore, FUNDC1, which is activated by PGAM5-mediated dephosphorylation cannot complete mitophagy to remove the damaged mitochondria because vinblastine prevents polymerization of microtubules, which inhibits fusion of autophagosomes with endosomes [37]. However, FUNDC1 can promote mitofission through recruitment of DRP1, which results in mitochondrial fragmentation that in turn facilitates mitochondrial permeabilization and apoptosis. Further dissecting the structural and molecular details of these important stress-dependent processes will provide better understanding of the life-or-death balance of the cell.

\section{Martials and methods}

\section{Cell culture and transfection}

HeLa cells were cultured in DMEM supplemented with $10 \%$ fetal bovine serum (Hyclone) and 1\% penicillinstreptomycin at $37^{\circ} \mathrm{C}$ under $5 \% \quad \mathrm{CO}_{2}$. Plasmids were transfected into cells with polyethylenimine according to the lab manufacturer's protocols.

\section{CRISPR/Cas9-mediated gene-knockout}

The CRISPR/Cas9 system was used to generate FUNDC1, $P G A M 5$, and $B C L-x L$ single and PGAM5/BCL- $x L$ double knockout HeLa cell lines. The oligonucleotide pairs used were as follows: $B C L-x L$ (5' - CACCGCAGGCGACG AGTTTGAACTG-3' and $5^{\prime}$-AAACCAGTTCAAACTCG TCGCCTGC-3'); PGAM5 (5' -CACCGCGCACGTTGAT CAGAGACAG-3' and 5' -AAAC GCAGGCGACGAGTT TGAACTGGTTTC- $\left.3^{\prime}\right)$; FUNDC1 (5' - CACCGCTGGCG GTATCATGGCGACC-3' and 5' - AAACGGTCGCCATG ATACCGCCAGC- $3^{\prime}$ ). Single clones were picked and expanded after selection with $2.5 \mu \mathrm{g} / \mathrm{ml}$. Knockout of the respective genes was confirmed by sequencing the edited genomic regions after amplification by PCR. The loss of the respective proteins was verified by immunoblotting with appropriate antibodies.

\section{Flow cytometry}

Cells were stained with $100 \mathrm{nM}$ MitoSox (ThermoFisher) for $15 \mathrm{~min}$ at $37^{\circ} \mathrm{C}$, washed 2 times with warm PBS, and then collected for analysis with flow cytometer (BD Calibur). Cells were collected and washed 2 times with warm PBS, then stained with FITC-Annexin V for 15 min at room temperature. Samples were stained with PI before analysis with a flow cytometer.

\section{Immunoblotting}

Cells treated with the indicated agents were harvested and lysed in lysis buffer $(150 \mathrm{mM} \mathrm{NaCl}, 20 \mathrm{mM}$ Tris, $\mathrm{pH} 7.4,1$ mM EGTA, $1 \mathrm{mM}$ EDTA, $1 \%$ SDS, $2.5 \mathrm{mM}$ sodium pyrophosphate, $1 \mathrm{mM} \mathrm{Na}_{3} \mathrm{VO}_{4}$, and $10 \mu \mathrm{M}$ PMSF). Cell lysates containing equivalent amounts of protein $(20 \mu \mathrm{g})$ were subjected to SDS-PAGE, and transferred to nitrocellulose membranes, which were blocked by $5 \%$ milk and probed with the indicated primary antibodies, followed by the appropriate HRP-conjugated secondary antibodies (KPL, 1912I). Immunoreactive bands were detected by a chemiluminescence kit (Engreen Biosystem, 29100) and visualized by a chemiluminescence imager (JUNYI). The following antibodies were used: Phospho-(Ser/Thr) (1:1000, Abcam, ab117253); BCL-xL (1:1000, Cell Signaling Technology, 2762); phospho-BCL-xL (pSer62) (1:1000, Sigma, SAB4504352); PGAM5 (1:1000, Abcam, ab131552); ACTIN (1:3000, Sigma, A5441); Tubulin (1:3000, MBL, PM054); TIMM23 (1:1000, BD Biosciences, 611222); TOMM20 (1:1000, BD Biosciences, 612278); COX IV (1:1000, Proteintech, 11242-1-AP); Cytochrome c (1:1000, BD Biosciences, 556433); MFN1 (1:1000, Abgent, ASC11815); Drp1(1:1000, BD Biosciences, 611113); phospho-DRP1 (pSer637) (1:1000, Cell Signaling Technology,4867S); BECN1 (1:1000, BD Biosciences, 612113); LC3 (1:1000, MBL, PM036); SQSTM1 (1:2000, MBL,PM045); BAX (1:1000, Santa Cruz, SC493); BAK (1:1000, Santa Cruz, SC-S17309); Cleaved Caspase-3 (1:1000, Cell Signaling Technology, 9664S); 
a

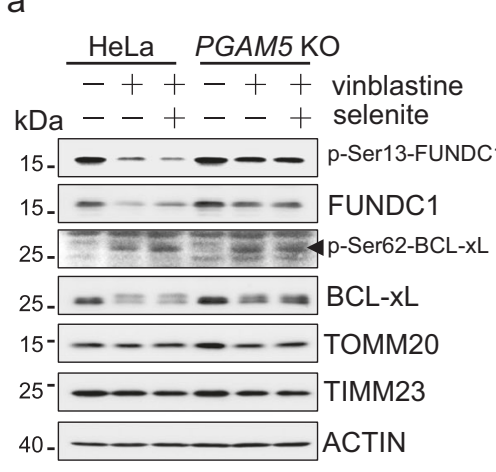

C

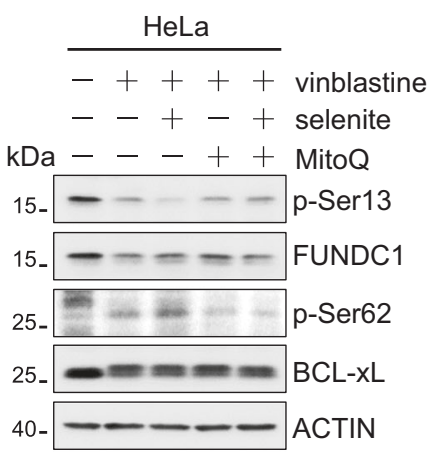

d

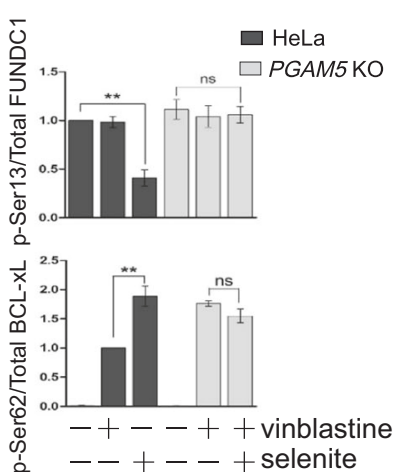

HeLa

$\overline{+++++}$ FLAG-BCL-XL

+++++ FLAG-FUNDC1

-++++ PGAM5-MYC

--+++ vinblastine

--++ selenite

$\mathrm{kDa}--\ldots+$ MitoQ

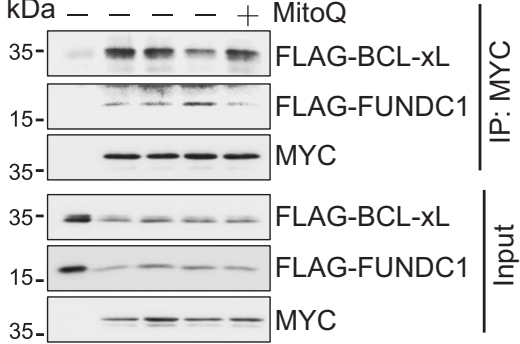

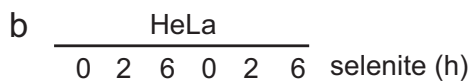

$\mathrm{kDa}--++++$ vinblastine

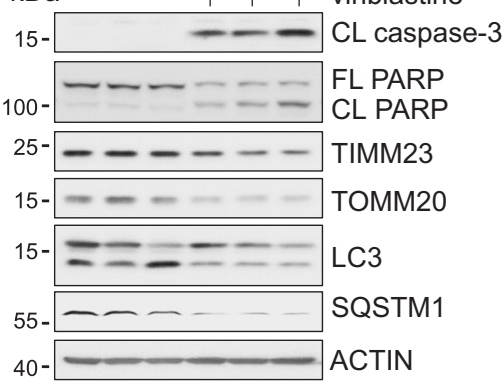

e $\frac{\text { HeLa }}{--+ \text { MitoQ }}$

$\mathrm{kDa}-++$ selenite

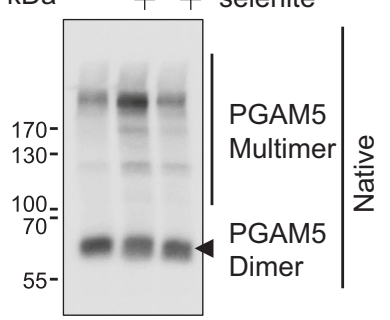

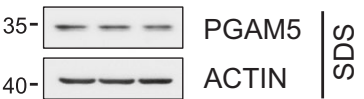

f
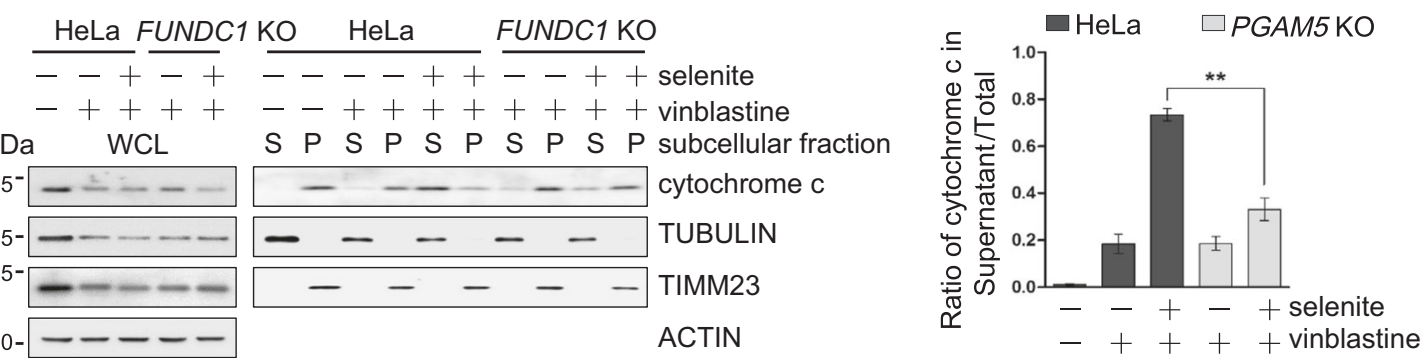

g

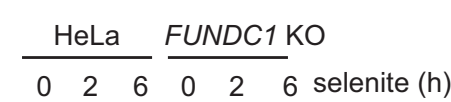
$\mathrm{kDa}++++++$ vinblastine $15-\square$ CL caspase-3
100- $\square$ PARP
$40-\square$ ACTIN
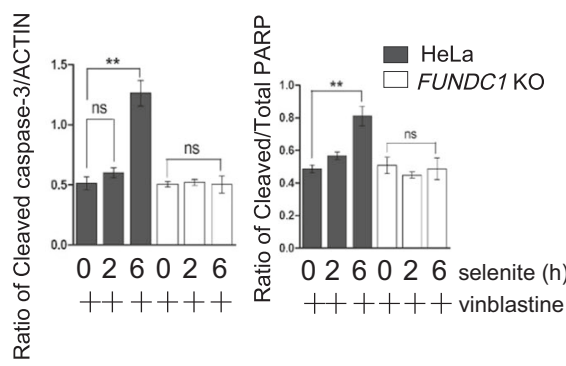

h

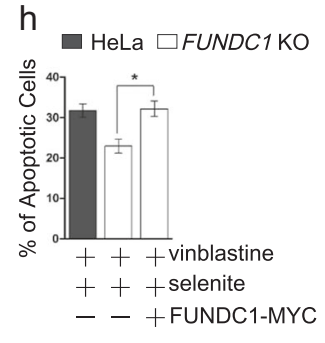

PARP (1:1000, Cell Signaling Technology, 9542); MYC (1:2000, Santa Cruz, B1115); FLAG (1:2000, Sigma, F1804); GFP (1:2000, Santa Cruz Biotechnology, A2115); and FUNDC1 (1:1000, AVIVA, ARP53280_P050). The anti-FUNDC1 (p-Ser13) polyclonal antibody $(1: 1,000)$ was affinity purified after immunizing rabbits with purified FUNDC1 phosphopeptides

\section{Immunoprecipitation}

Cells treated with the indicted agents were lysed with $0.5 \mathrm{ml}$ of lysis buffer ( $20 \mathrm{mM}$ Tris, $\mathrm{pH} 7.4,137 \mathrm{mM} \mathrm{NaCl}, 2 \mathrm{mM}$ EDTA, $10 \%$ glycerol, 1\% NP-40; Shanghai Sangon Biotech, A510110) plus protease inhibitor cocktail (Roche Applied Science, 11206893001) for $50 \mathrm{~min}$ on ice. After 
Fig. 6 Mitochondrial oxidative stress regulates dephosphorylation of BCL-xL and FUNDC1 by PGAM5 to promote apoptosis during mitotic arrest. a HeLa and PGAM5 knockout HeLa cells were treated with selenite ( $5 \mathrm{uM}, 2 \mathrm{~h})$ and/or vinblastine $(500 \mathrm{nM}, 24 \mathrm{~h})$, lysed and analyzed by immunoblotting with the indicated antibodies. Right: the ratio of p-Ser13 to total FUNDC1 or p-Ser62 to total BCL-xL were calculated from the respective band intensities in the respective blots measured by ImageJ, normalized to the values from untreated Hela cells or vinblastine-treated HeLa cells, respectively. Data are the mean \pm SEM of three experiments. ${ }^{*} p<0.01$. b HeLa cells were treated with $500 \mathrm{nM}$ vinblastine for $24 \mathrm{~h}$ in combination with $5 \mu \mathrm{M}$ selenite for the indicated times. After removal of selenite, the cells were cultured for an additional $24 \mathrm{~h}$, then lysed and analyzed by immunoblotting with the indicated antibodies. $\mathbf{c}$ HeLa cells were treated with vinblastine with or without MitoQ before addition of selenite as indicated, lysed and analyzed by immunoblotting with the indicated antibodies. d HeLa cells expressing FLAG-BCL-xL, FLAG-FUNDC1 and/or PGAM5-MYC were treated with vinblastine with or without MitoQ before addition of selenite as indicated, lysed and immunoprecipitated with anti-MYC antibody. The co-precipitated FLAGBCL-xL and FLAG-FUNDC1 were detected by immunoblotting with anti-FLAG antibody. e HeLa cells were treated with selenite for $2 \mathrm{~h}$, with MitoQ pretreatment if indicated, lysed, analyzed by native or denaturing SDS PAGE, then immunoblotted with the indicated antibodies. f HeLa and FUNDC1 knockout HeLa cells were treated with $500 \mathrm{nM}$ vinblastine for $36 \mathrm{~h}$ and $5 \mu \mathrm{M}$ selenite for $2 \mathrm{~h}$, lysed and fractionated. The cytochrome $\mathrm{c}$ in the heavy membrane pellet $(\mathrm{P})$ and supernatant (S) were detected by immunoblotting along with the cytoplasmic protein tubulin and the mitochondrial protein TIMM23. Right: the ratio of cytochrome $\mathrm{c}$ in supernatant to total cytochrome $\mathrm{c}$ were calculated. Data are the mean \pm SEM of three experiments. $* * p<$ 0.01. g HeLa and FUNDC1 knockout HeLa cells were treated with $500 \mathrm{nM}$ vinblastine for $48 \mathrm{~h}$ and $5 \mu \mathrm{M}$ selenite for the indicated times, lysed and analyzed by immunoblotting with the indicated antibodies. Right; the ratio of cleaved caspase- 3 to ACTIN and cleaved to total PARP were calculated from the respective band intensities measured by ImageJ. Data are the mean \pm SEM of three experiments. $* * p<0.01$. h HeLa or FUNDC1 knockout HeLa cells, transfected with FUNDC1MYC if indicated, were treated with vinblastine for $48 \mathrm{~h}$ and selenite $6 \mathrm{~h}$. After treatment, the cells were collected and stained with FITCannexin $\mathrm{V}$ and propidium iodide (PI), then analyzed by flow cytometry. The percentages of apoptotic cells (annexin V+/PI- and annexin $\mathrm{V}+/ \mathrm{PI}+$ ) were calculated. Data are the mean $\pm \mathrm{SEM}$ of three experiments. ${ }^{*} p<0.05$

centrifugation at $12,000 \mathrm{~g}$ for $10 \mathrm{~min}$, the lysates were incubated with $\mathrm{Ni}^{2+}$-chelating agarose beads (GE Healthcare) for 2 hours or specific antibody and then protein A/Gagarose beads (Abmart, A10001) overnight at $4{ }^{\circ} \mathrm{C}$. The beads were washed 5 times with lysis buffer, and the immune complexes were eluted with reducing SDS sample buffer for $5 \mathrm{~min}$ at $95^{\circ} \mathrm{C}$ and analyzed by SDS-PAGE and immunoblotting.

\section{Immunofluorescence microscopy}

After treatment, cells were fixed with freshly prepared $4 \%$ paraformaldehyde (Dingguo Changsheng Biotechnology) at $37^{\circ} \mathrm{C}$ for $20 \mathrm{~min}$, then permeabilized with $0.2 \%$ Triton $\mathrm{X}$ 100 (Shanghai Sangon Biotech, TB0198) at $4{ }^{\circ} \mathrm{C}$. After blocking in goat serum for $1 \mathrm{~h}$, cells were incubated with primary antibodies for $2 \mathrm{~h}$ at room temperature, washed with PBS, and stained with FITC- or CY5-conjugated secondary antibodies (Invitrogen) for $1 \mathrm{~h}$ at room temperature. Cell images were captured with a TCS SP5 Leica confocal microscope.

\section{Native PAGE}

Cells were lysed in RIPA lysis buffer $(50 \mathrm{mM}$ Tris-HCL, $\mathrm{pH} 7.4,150 \mathrm{mM} \mathrm{NaCl}, 0.5 \% \mathrm{NP} 40$ and PMSF). After centrifugation at $12,000 \mathrm{~g}$ for $10 \mathrm{~min}$, supernatant proteins were quantified and diluted with $2 \times$ native PAGE sample buffer (125 mM Tris-HCl, pH 6.8; 30\% glycerol; and $0.1 \%$ bromophenol blue). $15 \mu \mathrm{g}$ protein was used for nativePAGE using gels made without SDS. Gels were run with $25 \mathrm{mM}$ Tris, $\mathrm{pH} 8.4,192 \mathrm{mM}$ glycine and $1 \%$ deoxycholate (DOC) in a chamber. After electrophoresis, proteins were transferred to nitrocellulose membranes for immunoblotting.

\section{Phosphatase assay}

We were unable to purify recombinant full-length PGAM5 protein that contains a $\mathrm{N}$-terminal transmembrane sequence because it formed aggregates when expressed in Escherichia coli BL21 cells. We thus expressed a PGAM5 protein lacking the transmembrane sequence (His-PGAM5- $\Delta$ N21) and the phosphatase-dead mutant (His-PGAM5 (H105A)$\triangle \mathrm{N} 21)$ in Escherichia coli BL21 cells. Using Plasmids for His-PGAM5- $\Delta$ N21 and His-PGAM5 (H105A)- $\Delta$ N21 were from Dr. Xiaodong Wang's lab, and the proteins were expressed and purified from Escherichia coli BL21 cells.

$\mathrm{Ni}^{2+}$ beads were used as described above to enrich GFPHis-BCL-xL (pSer62). After three washes with phosphatase buffer (50 mM imidazole, pH 7.2, 0.2 mM EGTA, 0.02\% 2mecaptoethanol, and $0.1 \mathrm{mg} / \mathrm{ml} \mathrm{BSA}$ ), the beads were incubated with the recombinant His-tagged PGAM5 protein or the phosphatase-dead mutant (H105A) $(200 \mathrm{ng})$ at $30^{\circ} \mathrm{C}$ for 1 hour, and subjected to immunoblotting using antiphospho-BCL-xL (pSer62) antibody.

\section{Photocrosslinking}

The photocrosslinking method for studying interactions between BCL-2 family proteins has been described in detail. For this study $[38],\left[{ }^{35} \mathrm{~S}\right]$ Met-labeled BCL-xL proteins, each with a single $\mathrm{N}^{\varepsilon}$-(5-azido-2-nitrobenzoyl)-lysine ( $\varepsilon$ ANB-Lys) incorporated at a specific location, were synthesized from RNA encoding the corresponding single-Lys $\mathrm{BCL}-\mathrm{xL}$ mutants using an in vitro translation system [38]. $10 \mu \mathrm{l}$ of each resulting BCL-xL protein was incubated at $37^{\circ} \mathrm{C}$ for $1.5 \mathrm{~h}$ with $3 \mu \mathrm{M}$ purified His $_{6}$-tagged PGAM5 $\Delta$ N90 or $\Delta \mathrm{N} 54$ protein lacking the N-terminal 


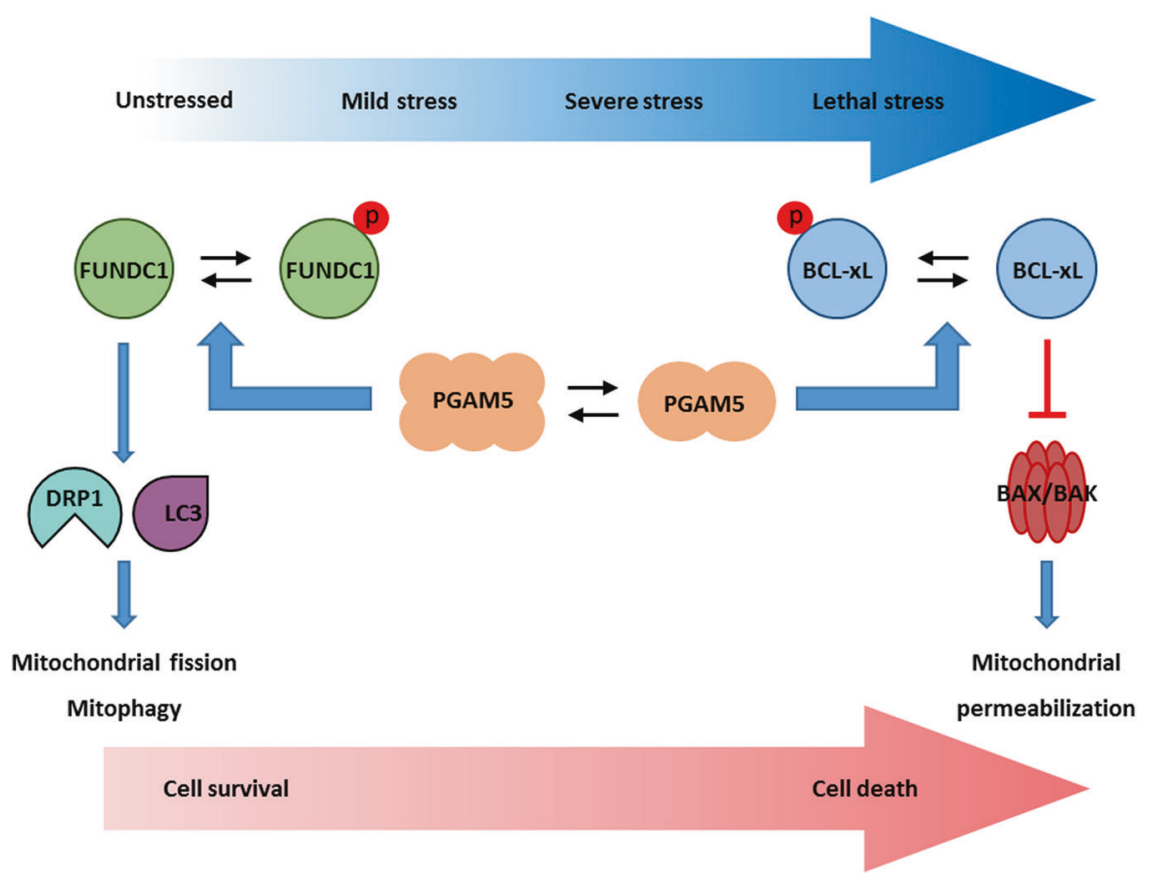

Fig. 7 Reciprocal interaction of PGAM5 with BCL-xL and FUNDC1 functions as a molecular switch to sense different stresses and generate appropriate responses to dictate mitochondrial and cellular fate. In unstressed cells, BCL-xL is not phosphorylated and binds the mitochondrial phosphatase PGAM5, whereas FUNDC1 is inhibited by phosphorylation. Severe stress such as the mitotic arrest induced by vinblastine is sensed by certain kinases that phosphorylate BCL-xL to reduce its interaction with BAX and BAK, and hence its anti-apoptotic function. PGAM5 in the dimeric state binds and dephosphorylates BCL-xL to reactivate its anti-apoptotic function. Mild stress such as mitochondrial ROS induced by selenite switches PGAM5 to the multimeric state, which cannot bind and reactivate BCL-xL to prevent mitochondrial damage. However, the multimeric PGAM5 binds and dephosphorylates FUNDC1 to activate mitochondrial fission and subsequent mitophagy to eliminate the damaged mitochondria, thus promoting cell survival. Lethal stress such as a combination of mitochondrial ROS and mitotic arrest promotes cell death by inactivating BCL-xL and blocking FUNDC1-dependent mitophagy but not mitochondrial fission

90 or 54 residues in a $20-\mu 1$ reaction containing buffer A. The samples were photolyzed to induce crosslinking via the ANB probe. The resulting photoadducts of the $\left[{ }^{35} \mathrm{~S}\right] \mathrm{Met}-$ labeled BCL-xL protein and the His ${ }_{6}$-tagged PGAM5 protein were enriched on $\mathrm{Ni}^{2+}$-nitrilotriacetic acid agarose, eluted and analyzed with reducing SDS-PAGE and phosphor-imaging. The following control reactions were carried out in parallel: the lysine-null (K0) BCL-xL control lacked the $\varepsilon$ ANB-Lys residue; the minus ANB control had BCL-xL with an unmodified Lys residue instead of the EANB-Lys; the minus light (hv) control had an ANB probe which was neutralized by DTT before photolysis; and the minus $6 \mathrm{H}-\mathrm{PGAM} 5 \Delta \mathrm{N} 90$ or $\Delta \mathrm{N} 54$ control lacked the $\mathrm{His}_{6}$ tagged protein. The $\mathrm{His}_{6}$-tagged PGAM5 $\Delta$ N90 and $\Delta$ N54 proteins were expressed and purified using plasmids from Dr. Apirat Chaikuad as described [27].

\section{Subcellular fractionation}

The cells were collected and resuspended in hypotonic buffer $(210 \mathrm{mM}$ sucrose, $70 \mathrm{mM}$ mannitol, $1 \mathrm{mM}$ EDTA, $1 \mathrm{mM}$ EGTA, $1.5 \mathrm{mM} \mathrm{MgCl}_{2}, 10 \mathrm{mM}$ HEPES, pH 7.2, $10 \mathrm{mM} \mathrm{KCl}$ ). After gentle homogenization with a Dounce homogenizer, the cell lysates were subjected to differential and gradient centrifugation $(800 \times g, 10 \mathrm{~min}$ and $8000 \times g$, $10 \mathrm{~min}$ ). The resulting membrane fractions were lysed by $1 \%$ SDS, and then analyzed together with the soluble fraction by immunoblotting.

\section{Statistical analysis}

Data were analyzed from three independent experiments and are shown as the mean \pm SEM. Statistical analyses were performed using Student's two-tailed $t$-test, with $p$ values $<$ 0.05 being considered significant. Significance levels relative to the controls are indicated by $* * *$ or $* * *$ for $p<$ $0.05 ; 0.01$ or 0.001 , respectively, in the figures. All analyses were performed with GraphPad Prism software.

Acknowledgements This research was supported by Grants 301520103904, 91754114, 31271529, 31671441 and 3192100015 from the Natural Science Foundation of China to YZ and QC, Grants 2016YFA0500201 and 2016YFA0100503 from the Ministry of Science and Technology of China to QC, Grant QYZDJ-SSW-SMC004 from the CAS Key Project of Frontier Science, 111 Project from the Ministry of Education and the State Administration of Foreign Experts Affairs of the People's Republic of China (B08011), Grant 
R01GM062964 from the United States National Institutes of Health and Grant HR16-026 from the Oklahoma Center for the Advancement of Science and Technology to JL, and by an Institutional Development Award from the National Institute of General Medical Sciences of the National Institutes of Health under grant number P20GM103640.

Author contributions $\mathrm{KM}$ conceived and designed the experiments with YZ, JL and QC; KM performed most of the experiments and data analysis with assistance from RC, HC, MC, TZ, BC, CM and QL. ZZ performed the photocrosslinking experiment. KM, QC, JL and YZ wrote the manuscript. All authors provided intellectual input to the manuscript.

\section{Compliance with ethical standards}

Conflict of interest The authors declare that they have no conflict of interest.

Publisher's note: Springer Nature remains neutral with regard to jurisdictional claims in published maps and institutional affiliations.

\section{References}

1. Galluzzi L, Kepp O, Trojel-Hansen C, Kroemer G. Mitochondrial control of cellular life, stress, and death. Circ Res. 2012;111:1198-207.

2. Circu ML, Aw TY. Reactive oxygen species, cellular redox systems, and apoptosis. Free Radic Biol Med. 2010;48:749-62.

3. Wallace DC. A mitochondrial paradigm of metabolic and degenerative diseases, aging, and cancer: a dawn for evolutionary medicine. Annu Rev Genet. 2005;39:359-407.

4. Kalkavan H, Green DR. MOMP, cell suicide as a BCL-2 family business. Cell Death Differ. 2018;25:46-55.

5. Palikaras K, Lionaki E, Tavernarakis N. Mechanisms of mitophagy in cellular homeostasis, physiology and pathology. Nat Cell Biol. 2018;20:1013-22.

6. Novak I, Kirkin V, McEwan DG, Zhang J, Wild P, Rozenknop A, et al. Nix is a selective autophagy receptor for mitochondrial clearance. EMBO Rep. 2010;11:45-51.

7. Liu L, Feng D, Chen G, Chen M, Zheng Q, Song P, et al. Mitochondrial outer-membrane protein FUNDC1 mediates hypoxia-induced mitophagy in mammalian cells. Nat Cell Biol. 2012;14:177-85.

8. Wei Y, Chiang WC, Sumpter R Jr., Mishra P, Levine B. Prohibitin 2 Is an Inner Mitochondrial Membrane Mitophagy Receptor. Cell. 2017;168:224-38 e210.

9. Wang Z, Jiang H, Chen S, Du F, Wang X. The mitochondrial phosphatase PGAM5 functions at the convergence point of multiple necrotic death pathways. Cell 2012;148:228-43.

10. Lenhausen AM, Wilkinson AS, Lewis EM, Dailey KM, Scott AJ, Khan S, et al. Apoptosis inducing factor binding protein PGAM5 triggers mitophagic cell death that is inhibited by the ubiquitin ligase activity of X-linked inhibitor of apoptosis. Biochemistry. 2016;55:3285-302.

11. Imai Y, Kanao T, Sawada T, Kobayashi Y, Moriwaki Y, Ishida Y, et al. The loss of PGAM5 suppresses the mitochondrial degeneration caused by inactivation of PINK1 in Drosophila. PLoS Genet. 2010;6:e1001229.

12. Sekine S, Yao A, Hattori K, Sugawara S, Naguro I, Koike M, et al. The ablation of mitochondrial protein phosphatase Pgam5 confers resistance against metabolic stress. EBioMedicine. 2016;5:82-92.

13. Lu W, Karuppagounder SS, Springer DA, Allen MD, Zheng L, Chao B, et al. Genetic deficiency of the mitochondrial protein
PGAM5 causes a Parkinson's-like movement disorder. Nat Commun. 2014;5:4930.

14. Chen G, Han Z, Feng D, Chen Y, Chen L, Wu H, et al. A regulatory signaling loop comprising the PGAM5 phosphatase and CK2 controls receptor-mediated mitophagy. Mol cell. 2014;54:362-77.

15. Lo SC, Hannink M. PGAM5, a Bcl-XL-interacting protein, is a novel substrate for the redox-regulated Keap1-dependent ubiquitin ligase complex. J Biol Chem. 2006;281:37893-903.

16. Wang J, Beauchemin M, Bertrand R. Bcl-xL phosphorylation at Ser49 by polo kinase 3 during cell cycle progression and checkpoints. Cell Signal. 2011;23:2030-8.

17. Kharbanda S, Saxena S, Yoshida K, Pandey P, Kaneki M, Wang $\mathrm{Q}$, et al. Translocation of SAPK/JNK to mitochondria and interaction with $\mathrm{Bcl}-\mathrm{x}(\mathrm{L})$ in response to DNA damage. J Biol Chem. 2000;275:322-7.

18. Megyesi J, Tarcsafalvi A, Seng N, Hodeify R, Price PM. Cdk2 phosphorylation of Bcl-xL after stress converts it to a proapoptotic protein mimicking Bax/Bak. Cell Death Discov. 2016;2: pii: 15066.

19. Del Re DP, Matsuda T, Zhai P, Maejima Y, Jain MR, Liu T, et al Mst1 promotes cardiac myocyte apoptosis through phosphorylation and inhibition of Bcl-xL. Mol Cell. 2014;54:639-50.

20. Wilkins JM, McConnell C, Tipton PA, Hannink M. A conserved motif mediates both multimer formation and allosteric activation of phosphoglycerate mutase 5. J Biol Chem. 2014;289:25137-48.

21. Terrano DT, Upreti M, Chambers TC. Cyclin-dependent kinase 1mediated $\mathrm{Bcl}-\mathrm{xL} / \mathrm{Bcl}-2$ phosphorylation acts as a functional link coupling mitotic arrest and apoptosis. Mol Cell Biol. 2010;30:640-56.

22. Fan M, Goodwin M, Vu T, Brantley-Finley C, Gaarde WA, Chambers TC. Vinblastine-induced phosphorylation of Bcl-2 and $\mathrm{Bcl}-\mathrm{XL}$ is mediated by JNK and occurs in parallel with inactivation of the Raf-1/MEK/ERK cascade. J Biol Chem. 2000;275:29980-5.

23. Arena G, Gelmetti V, Torosantucci L, Vignone D, Lamorte G, De Rosa P, et al. PINK1 protects against cell death induced by mitochondrial depolarization, by phosphorylating Bcl-xL and impairing its pro-apoptotic cleavage. Cell Death Differ. 2013;20:920-30.

24. Follis AV, Llambi F, Kalkavan H, Yao Y, Phillips AH, Park CG, et al. Regulation of apoptosis by an intrinsically disordered region of Bcl-xL. Nat Chem Biol. 2018;14:458-65.

25. Xu W, Jing L, Wang Q, Lin CC, Chen X, Diao J, et al. BaxPGAM5L-Drp1 complex is required for intrinsic apoptosis execution. Oncotarget. 2015;6:30017-34.

26. Hawk MA, Gorsuch CL, Fagan P, Lee C, Kim SE, Hamann JC, et al. RIPK1-mediated induction of mitophagy compromises the viability of extracellular-matrix-detached cells. Nat Cell Biol. 2018;20:272-84.

27. Chaikuad A, Filippakopoulos P, Marcsisin SR, Picaud S, Schroder M, Sekine S, et al. Structures of PGAM5 Provide Insight into Active Site Plasticity and Multimeric Assembly. Structure. 2017;25:1089-99 e1083.

28. Hammond PW, Alpin J, Rise CE, Wright M, Kreider BL. In vitro selection and characterization of $\mathrm{Bcl}-\mathrm{X}(\mathrm{L})$-binding proteins from a mix of tissue-specific mRNA display libraries. J Biol Chem. 2001;276:20898-906.

29. Wu H, Xue D, Chen G, Han Z, Huang L, Zhu C, et al. The BCL2L1 and PGAM5 axis defines hypoxia-induced receptormediated mitophagy. Autophagy. 2014;10:1712-25.

30. Ding J, Mooers BH, Zhang Z, Kale J, Falcone D, McNichol J, et al. After embedding in membranes antiapoptotic Bcl-XL protein binds both Bcl-2 homology region 3 and helix 1 of proapoptotic Bax protein to inhibit apoptotic mitochondrial permeabilization. $\mathbf{J}$ Biol Chem. 2014;289:11873-96. 
31. Ruiz K, Thaker TM, Agnew C, Miller-Vedam L, Trenker R, Herrera $\mathrm{C}$, et al. Functional role of PGAM5 multimeric assemblies and their polymerization into filaments. Nat Commun. 2019;10:531.

32. Chen M, Chen Z, Wang Y, Tan Z, Zhu C, Li Y, et al. Mitophagy receptor FUNDC1 regulates mitochondrial dynamics and mitophagy. Autophagy. 2016;12:689-702.

33. Huang F, Nie C, Yang Y, Yue W, Ren Y, Shang Y, et al. Selenite induces redox-dependent Bax activation and apoptosis in colorectal cancer cells. Free Radic Biol Med. 2009;46:1186-96.

34. Estaquier J, Arnoult D. Inhibiting Drp1-mediated mitochondrial fission selectively prevents the release of cytochrome c during apoptosis. Cell Death Differ. 2007;14:1086-94.

35. Nie C, Tian C, Zhao L, Petit PX, Mehrpour M, Chen Q. Cysteine 62 of Bax is critical for its conformational activation and its proapoptotic activity in response to $\mathrm{H} 2 \mathrm{O} 2$-induced apoptosis. J Biol Chem. 2008;283:15359-69.

36. Vermeij WP, Backendorf C. Reactive oxygen species (ROS) protection via cysteine oxidation in the epidermal cornified cell envelope. Methods Mol Biol. 2014;1195:157-69.

37. Kochl R, Hu XW, Chan EY, Tooze SA. Microtubules facilitate autophagosome formation and fusion of autophagosomes with endosomes. Traffic. 2006;7:129-45.

38. Lin J, Johnson AE, Zhang Z. Photocrosslinking Approach to Investigate Protein Interactions in the BCL-2 Family. Methods Mol Biol. 2019;1877:131-49.

39. Muchmore SW, Sattler M, Liang H, Meadows RP, Harlan JE, Yoon HS, et al. X-ray and NMR structure of human Bcl-xL, an inhibitor of programmed cell death. Nature. 1996; $381: 335-41$. 